\title{
Heterotic complex structure moduli stabilization for elliptically fibered Calabi-Yau manifolds
}

\author{
Wei $\mathrm{Cui}^{a}$ and Mohsen Karkheiran ${ }^{b}$ \\ ${ }^{a}$ Department of Physics, Robeson Hall, Virginia Tech, \\ 850 West Campus Dr, Blacksburg, VA 24061, U.S.A. \\ ${ }^{b}$ Center for Theoretical Physics of the Universe, Institute for Basic Science (IBS), \\ 55 Expo-Ro, Daejeon, 34126, Korea \\ E-mail: cwei@vt.edu, mohsenkar@ibs.re.kr
}

ABSTRACT: Holomorphicity of vector bundles can stabilize complex structure moduli of a Calabi-Yau threefold in $N=1$ supersymmetric heterotic compactifications. In principle, the Atiyah class determines the stabilized moduli. In this paper, we study how this mechanism works in the context of elliptically fibered Calabi-Yau manifolds where the complex structure moduli space contains two kinds of moduli, those from the base and those from the fibration. Defining the bundle with spectral data, we find three types of situations when bundles' holomorphicity depends on algebraic cycles exist only for special loci in the complex structure moduli, which allows us to stabilize both of these two moduli. We present concrete examples for each type and develop practical tools to analyze the stabilized moduli. Finally, by checking the holomorphicity of the four-flux and/or local Higgs bundle data in F-theory, we briefly study the dual complex structure moduli stabilization scenarios.

KEYwords: Superstring Vacua, Superstrings and Heterotic Strings

ArXiv EPrint: 2011.14304 


\section{Contents}

1 Introduction 1

2 The Atiyah class 3

2.1 A rank two extension bundle 5

3 Complex structure moduli stabilization mechanisms $\quad 8$

3.1 Type I: "jumping" extension group 9

3.2 Type II: "jumping" Picard group 12

$\begin{array}{ll}3.3 & \text { Type III: emergent algebraic cycle } \\ \end{array}$

4 Discussion and comments on F-theory dual 18

5 Conclusion $\quad 21$

A Complex structure moduli space of elliptically fibered Calabi-Yau threefold

B Type II and type III Fourier-Mukai calculations 24

$\begin{array}{ll}\text { C Computation details of "jumping" ext } & 25\end{array}$

D Practical approach to identify reducible deformations 26

\section{Introduction}

Compactification of $E_{8} \times E_{8}$ heterotic string [1,2] on a Calabi-Yau threefold with a vector bundle is a promising approach to derive the Standard Model of particle physics in the four-dimensional spacetime [3-11]. To preserve supersymmetry, the bundle should be holomorphic, slope zero, and poly-stable. Fluctuations of the complex structure and Kähler form of the base manifold and fluctuations of the bundle appear as moduli particles in the effective theory. Holomorphicity of the bundle has been used to stabilize the complex structure moduli in these models [12-16]. The idea is that only some of the deformations of the complex structure are consistent with the supersymmetry. The others will force the bundle to become non-holomorphic. This will generate non-trivial F-terms for such deformations in the effective theory, and the complex structure moduli corresponding to them are stabilized. As shown in [14], this mechanism, together with a similar one due to bundle poly-stability [17-21] is an effective way to stabilize geometric moduli in heterotic Calabi-Yau compactification.

Deformations of the complex structure-preserving bundle holomorphy are described by the Atiyah class [22]. The computation of it is, in general, challenging. However, for 
holomorphic bundles in which the necessary ingredients in the construction have a complex structure dependence, these deformations can be calculated efficiently by a "bottom-up" approach. For example, extension bundles [5] are defined by specifying an element in the Ext group. As shown in $[12,13]$, the Ext group jumps in dimension for a special choice of the complex structure. This signals that some of the complex structure moduli are stabilized locally over the particular locus in moduli space. Instead of the Atiyah class, the stabilized moduli can be identified quickly by a computation of "jumping" Ext. As a result, the "bottom-up" approaches are equivalent to the calculation of the Atiyah class and are efficient tools for the complex structure moduli stabilization.

In this work, we will focus on Calabi-Yau threefolds with an elliptic fibration ${ }^{1}[23-$ 30], and study how its complex structure moduli can be stabilized by bundle holomorphy. Bundles on an elliptically fibered Calabi-Yau threefold can be constructed by spectral data [31-34], which can be mapped to the extension or monad bundles by a Fourier-Mukai transformation. The reason to work with the spectral cover bundle is that poly-stability, a difficult property to check for other constructions, is straightforward to study in terms of spectral data, at least as long as the spectral cover is smooth. Besides, since the spectral data specify the Heterotic models, the Heterotic model's moduli stabilization process can be translated to an associated process in the dual F-theory model. Hence the relation with moduli stabilization scenarios in F-theory/type II models will be manifest.

Complex structure moduli of an elliptically fibered Calabi-Yau threefold $X$ can be either the complex structure moduli of the base $B$ or the complex structure moduli of the fibration $F$. As we will see later, both of them can be stabilized using holomorphic bundles. Specifically, we find three types of approaches to do that but, from the perspective of spectral cover bundles, they all correspond to emergent algebraic cycles in $X$. In more technical terms, we look for the Noether-Lefschetz loci in the complex structure moduli and specify the spectral data so that the associated vector bundle stays holomorphic only on these loci. For type I case, we consider a SU(2) extension bundle with Ext group jumping on a special locus in complex structure moduli space of $B$. One can observe that variations of the complex structure away from the special locus in moduli space will make the algebraic curve disappear. The spectral cover is not algebraic anymore. Hence the corresponding vector bundle becomes non-holomorphic, and the associated complex structure moduli of $B$ are stabilized. We will get some insight into how the stabilized moduli can be identified by studying the spectral data directly.

In the type II approach, we find that the Picard group of $B$ jumps when the complex structure of the base takes some "special" form. Suppose the holomorphic bundle is defined with the new algebraic cycles in the "jumping" Picard group. Then, variations of the complex structure that does not preserve this "special" form of complex structure will make the bundle non-holomorphic, and the corresponding moduli are stabilized. Besides the base's complex structure moduli, holomorphic bundles can also stabilize the moduli of the fibration. The type III approach is similar to the type II one, in which taking a

\footnotetext{
${ }^{1}$ The Calabi-Yau manifolds considered here are all in the Weierstrass form. This paper's ideas will be valid for any elliptic fibration, as long as there is at least one holomorphic section. However, when all of the sections are rational, one will need more careful analysis [33, 34].
} 
"special" complex structure gives new algebraic cycles in the base. The difference is that this time, the algebraic curves appear in the total space of the fibration. Suppose the spectral data are defined in terms of these emergent algebraic cycles. Then, variations of the complex structure away from the "special" form will make algebraic cycles nonalgebraic, the bundle becomes non-holomorphic, and the associated moduli are stabilized. Notice that the last two approaches do not require the bundle to be constructed as an extension and are applicable to more general bundles. We give explicit examples for all three approaches. Like "jumping" Ext, we develop practical tools to identify variations of the complex structure that does not preserve the "special" form used above and thus obtain the stabilized moduli in type II and type III approaches. ${ }^{2}$

The image of these moduli stabilization scenarios is also interesting. In the type I example, the flux derived from the spectral line bundle is responsible for the moduli stabilization. Finally, the four-flux in type II example is the horizontal algebraic four-flux constructed in [35]. A recent work on complex structure moduli stabilization also used the idea of algebraic cycles is discussed in [36].

This paper is organized as follows: in section 2, we review how a vector bundle's holomorphicity can be used for complex structure moduli stabilization in Calabi-Yau compactification. Specially we review the Atiyah class and illustrate it with a SU(2) extension bundle. In section 3, we introduce three approaches to stabilize the complex structure moduli of an elliptically fibered Calabi-Yau threefold with holomorphic bundles in spectral cover construction. In section 4 , we discuss how these approaches in heterotic compactification manifest themselves in F-theory. In section 5, we conclude our work. Some of the technical discussions are presented in the appendices.

\section{The Atiyah class}

Let $X$ be a Calabi-Yau threefold with a vector bundle $V$. To preserve $N=1$ supersymmetry, the bundle should have a connection satisfying the following (zero slope) Hermitian Yang-Mills equations,

$$
F_{a b}=F_{\bar{a} \bar{b}}=0, g^{a \bar{b}} F_{a \bar{b}}=0,
$$

where $F$ is the gauge field strength associated with that connection and $a$ and $\bar{b}$ are holomorphic and anti-holomorphic indices on the Calabi-Yau manifold. The first equation in (2.1) implies that the bundle is holomorphic [37]. It is the property that will be used for moduli stabilization. The second equation in (2.1) means that the bundle is poly-stable [38, 39]. We will always keep $V$ poly-stable when the complex structure of $X$ is varied. As shown in $[12,13]$, one can do that as long as the bundle is holomorphic.

We start with a supersymmetric vacuum where the initial Kähler, complex structure, and bundle moduli are chosen to obey the Hermitian Yang-Mills equation in (2.1). Notice that the initial objects will be denoted with a superscript "(0)" and their deformation

\footnotetext{
${ }^{2}$ It is interesting to see how many of the moduli fields can be stabilized in the phenomenologically interesting models using the methods presented in this paper. However, we only explain the general ideas here and leave these questions for a future project.
} 
will be denoted with a $\delta$ in front. From the deformation theory of compact complex manifolds [22, 40-43], the space of simultaneous holomorphic deformations of $X$ and $V$ is measured by $H^{1}(\mathcal{Q})$ with $Q$ satisfying the Atiyah sequence

$$
0 \rightarrow V \otimes V^{\vee} \rightarrow \mathcal{Q} \stackrel{\pi}{\rightarrow} T X \rightarrow 0
$$

The extension class is

$$
\alpha=\left[F^{(0) 1,1}\right] \in H^{1}\left(V \otimes V^{\vee} \otimes T X^{\vee}\right)
$$

the $(1,1)$ component of the initial field strength $F^{(0)}$ of the background, referred to as the "Atiyah class" [22]. From the long exact sequence of (2.2), we have

$$
0 \rightarrow H^{1}\left(V \otimes V^{\vee}\right) \rightarrow H^{1}(\mathcal{Q}) \stackrel{d \pi}{\rightarrow} H^{1}(T X) \stackrel{\alpha}{\rightarrow} H^{2}\left(V \otimes V^{\vee}\right) \rightarrow \ldots
$$

The simultaneous holomorphic deformations space $H^{1}(\mathcal{Q})$ is given by

$$
H^{1}(\mathcal{Q})=H^{1}\left(V \otimes V^{\vee}\right) \oplus \operatorname{Ker}(\alpha) .
$$

Notice that we have used the fact that $H^{0}(T X)=0$ for the stable bundle $T X$ in (2.4).

As we can see in (2.5), all bundle moduli in $H^{1}\left(V \otimes V^{\vee}\right)$ belong to $H^{1}(\mathcal{Q})$ while for complex structure moduli, only those that are in the kernel of the Atiyah class are elements of $H^{1}(\mathcal{Q})$ and should be regarded as the "true" moduli in the effective theory. The other ones have non-zero images in $H^{2}\left(V \otimes V^{\vee}\right)$, which lead to non-vanishing field strengths $F^{0,2}$ in the background and make $V$ non-holomorphic. Thus, variations of the complex structure along those directions are stabilized by non-trivial F-terms in superpotential. The number of stabilized moduli is counted by the dimension of $\operatorname{Im}(\alpha)$. From the sequence in (2.4), it is bounded by $h^{2}\left(V \otimes V^{\vee}\right)$, so there are at most $h^{2}\left(V \otimes V^{\vee}\right)$ complex structure moduli that can be stabilized by the holomorphic bundle.

The above mechanism can be made more precise if we write the elements of $H^{1}(T X)$ explicitly as $\nu=\delta \mathfrak{z}^{I} v_{I \bar{a}}^{c}$ where $v_{I}$ are tangent bundle valued harmonic one-forms and $\delta \mathfrak{z}^{I}$ are variations of the complex structure moduli $\mathfrak{z}^{I}$. By the sequence (2.4), elements in the kernel of Atiyah class should satisfy,

$$
\delta_{\mathfrak{z}}^{I} v_{I[\bar{a}}^{c} F_{|c| \bar{b}]}^{(0)}=D_{[\bar{a}}^{(0)} \Lambda_{\bar{b}]}
$$

where $\Lambda$ is a bundle-valued one-form and $D^{(0)}$ is the covariant derivative with respect to the initial connection $A^{(0)}$. Notice that the right hand side of the equation above is an exact 2-form in $H^{2}\left(X, V \otimes V^{\vee}\right)$. In fact, the bundle-valued one-form $\Lambda$ can be understood as deformation of the connection $\Lambda_{\bar{b}}=\delta A_{\bar{b}}$. Thus, for deformations of complex structure $\delta \mathfrak{z}^{I}$ that are in $\operatorname{Ker}(\alpha)$, there exist a deformation of the connection $\delta A$ such that the equation (2.6) is satisfied and $V$ can remain holomorphic. However, other deformations will make $V$ non-holomorphic, and the associated moduli are stabilized.

In conclusion, with Atiyah class defined in (2.3), we can determine which deformations of the complex structure in $H^{1}(T X)$ can keep $V$ holomorphic and which are not. The number of these independent deformations are given by the dimension of $\operatorname{Ker}(\alpha)$ and $\operatorname{Im}(\alpha)$ respectively. In general, they can be computed directly from the long exact sequence 
in (2.4) known as the "top-down" approach. However, for some holomorphic bundles defined properly only with some special complex structure, there is a quick way to determine the $\operatorname{Ker}(\alpha)$ and $\operatorname{Im}(\alpha)$. This "bottom-up" approach is equivalent ${ }^{3}$ to the direct computation of $H^{1}(\mathcal{Q})$ but is much easier computationally [13]. We will illustrate this method with a simple extension bundle in the following subsection.

\subsection{A rank two extension bundle}

We assume that the Calabi-Yau threefold $X$ is defined as a hypersurface in a product of projective spaces $\mathcal{A}=\mathbb{P}^{n_{1}} \times \ldots \times \mathbb{P}^{n_{m}}$. We will further ask that it is "favorable," i.e. the Picard group of $X$ is spanned by the restriction to $X$ of ambient divisors, $D_{i}$, associated with the hyperplane class in $\mathbb{P}^{n_{i}}$. The defining polynomial of $X$ is given by $p_{0} \in H^{0}(\mathcal{A}, N)$. Here $N$ is the normal bundle of the hypersurface. Notice that the defining polynomial $p_{0}$ is a redundant description of the complex structure. The independent ones can be obtained from the computation of $H^{1}(X, T X)$. We will discuss this in section 3.

Consider a rank two bundle $V$ defined as an extension of a line bundle $L$ and its dual

$$
0 \rightarrow L \rightarrow V \rightarrow L^{\vee} \rightarrow 0
$$

with the extension class $\phi \in E x t^{1}\left(L^{\vee}, L\right)=H^{1}\left(L^{2}\right)$. If $\phi \neq 0$, the extension is nontrivial and the bundle defined is a $\mathrm{SU}(2)$ indecomposable bundle, otherwise, the extension is trivial, and the bundle splits into $V=L \oplus L^{\vee}$. However, the non-trivial extension does not always exist on $X$ because the Ext group $H^{1}\left(L^{2}\right)$ that the extension class takes value in depends on the choice of complex structure. We will be interested in a situation where $H^{1}\left(L^{2}\right)$ is non-trivial only for some special complex structure of $X$ and $H^{1}\left(L^{2}\right)=0$ otherwise. Thus, the indecomposable $\mathrm{SU}(2)$ bundle is well-defined on a special locus in the moduli space, and it splits when the complex structure is deformed away from this locus. This setting will play a key role in the moduli stabilization discussed later.

Let's assume that the initial complex structure is chosen such that the $H^{1}\left(L^{2}\right)$ jumps in dimension and the bundle defined in (2.7) is an indecomposable $\mathrm{SU}(2)$ bundle. At the same time, one needs to choose the initial Kähler form $\omega$ of $X$ so that $V$ is poly-stable. Although poly-stability is in general difficult to check, for this simple extension bundle, it can be determined relatively easier ${ }^{4}$ by the following criterion [19]. $V$ is poly-stable if the Kähler form $\omega$ satisfies $\mu(L)<0$ where $\mu(L)$ is the slope of $L$ defined by

$$
\mu(L)=\frac{1}{\operatorname{rank}(V)} \int_{X} c_{1}(L) \wedge \omega \wedge \omega .
$$

With this condition, we will fix the initial Kähler moduli of $X$ so that $V$ is poly-stable in this example.

Now, let's consider the complex structure deformations with respect to this background. From the equation (2.3), deformations that are consistent with the holomorphic

\footnotetext{
${ }^{3}$ For the rank two vector bundles constructed as an extension of a line bundle and its dual, it is proved in [13] that the Atiyah class computation is rigorously equivalent to an analysis of "Jumping" of Ext group. This is an example of the "bottom-up" approach.

${ }^{4}$ It have been shown in [19], it is a sufficient condition for $V$ being poly-stable.
} 
bundle is measured by the kernel of the Atiyah class $\alpha \in H^{1}\left(V \otimes V^{\vee} \otimes T X^{\vee}\right)$. For this example, it can be shown [19] that $\alpha$ is an element of $H^{1}\left(X, L^{2} \otimes T X^{\vee}\right)$, a subset of $H^{1}\left(V \otimes V^{\vee} \otimes T X^{\vee}\right)$. Thus, the part of the sequence in (2.4) containing $\alpha$ becomes

$$
\alpha: H^{1}(X, T X) \rightarrow H^{2}\left(X, L^{2}\right) .
$$

By Bott-Borel-Weil Theorem [37], one can express the source $H^{1}(X, T X)$ and target $H^{2}\left(X, L^{2}\right)$ in terms of homogeneous polynomials. The kernel of the map $\alpha$ can be found by a direct computation of these polynomials. However, the non-trivial extension does not always exist on $X$ because the Ext group $H^{1}\left(L^{2}\right)$ that the extension class takes value in depends on the choice of the complex structure.

The idea of the "bottom-up" approach [19] is that instead of the direct calculation of $\operatorname{Ker}(\alpha)$ outlined above, the same information can be obtained by studying the "jumping" of Ext group $H^{1}\left(X, L^{2}\right)$. Specifically, recall that the indecomposable bundle $V$ considered here is defined on the special locus in the moduli space where Ext group $H^{1}\left(X, L^{2}\right)$ jumps. Consider the deformation of complex structure $p_{0} \rightarrow p_{0}+\delta p$. If $\delta P$ is within the special locus of moduli space, the $V$ is still indecomposable and holomorphic. However, if $\delta P$ is generic, the Ext group becomes $H^{1}\left(X, L^{2}\right)=0$. It seems that in this case, the only choice for extension class is $\phi=0$ and the bundle becomes a direct sum $L \oplus L^{\vee}$. In fact, the extension class $\phi \in H^{1}\left(X, L^{2}\right)$, does not change when we vary the complex structure moduli. Instead, $\phi$ is no longer a closed $(0,1)$-form with respect to the new complex structure. It means that $V$ after generic deformations $\delta p$ is not holomorphic anymore. In this example, we find that deformations of the complex structure that make the Ext group "jumping" are the same deformations that keep $V$ holomorphic. In contrast, deformations of the complex structure that leads to the trivial Ext group correspond to ones that make $V$ non-holomorphic. Thus, we see clearly that the calculation of "jumping" Ext is equivalent to the Atiyah computation.

Next, let's briefly describe how to identify the special locus where the Ext group $H^{1}\left(X, L^{2}\right)$ jumps in the moduli space. By the Koszul sequence, [37], the line bundle $L^{2}$ on $X$ can be expressed in terms of bundles on the ambient space $\mathcal{A}$. For the manifold considered here, it is

$$
0 \rightarrow N^{\vee} \otimes L_{\mathcal{A}}^{2} \stackrel{p_{0}}{\rightarrow} L_{\mathcal{A}}^{2} \rightarrow L^{2} \rightarrow 0,
$$

where $N^{\vee}$ is the dual of the normal bundle of $X$. The cohomology $H^{1}\left(X, L^{2}\right)$ can be computed via the associated long exact sequence

$$
0 \rightarrow H^{1}\left(X, L^{2}\right) \rightarrow H^{2}\left(\mathcal{A}, N^{\vee} \otimes L_{\mathcal{A}}^{2}\right) \stackrel{p_{0}}{\rightarrow} H^{2}\left(\mathcal{A}, L_{\mathcal{A}}^{2}\right) \rightarrow H^{2}\left(X, L^{2}\right) \rightarrow 0 .
$$

Here $p_{0}$ is the defining polynomial of $X$. Thus, we have $H^{1}\left(X, L^{2}\right)=\operatorname{Ker}\left(p_{0}\right)$ and $H^{1}\left(X, L^{\vee 2}\right)=\operatorname{Coker}\left(p_{0}\right)$. For generic defining polynomials, both $\operatorname{Ker}\left(p_{0}\right)$ and $\operatorname{Coker}\left(p_{0}\right)$ are trivial. While for some special choice of $p_{0}$, they can "jump" together so that the index $\operatorname{Ind}\left(L^{2}\right)=-h^{1}\left(X, L^{2}\right)+h^{2}\left(X, L^{2}\right)$ is preserved [13]. Note that, for the variation of the complex structure $p \rightarrow p_{0}+\delta p$, the number of independent variations that make $h^{1}\left(X, L^{2}\right)$ jumps is the same as the dimension of $\operatorname{Ker}(\alpha)$ computed from the equation (2.9) while the other variations of complex structure with $h^{1}\left(X, L^{2}\right)=0$ corresponds to $\operatorname{Im}(\alpha)$ in the Atiyah computation. 
This moduli stabilization mechanism can also be understood in the low-energy effective theory. Let $\mathfrak{z}_{0}$ be the initial complex structure moduli chosen within the special locus of moduli space where Ext group of the bundle defined in (2.7) jumps. The non-trivial extension defines a $\mathrm{SU}(2)$ bundle. The low-energy gauge group is $E_{7}$. However, if the extension class is trivial, $V$ splits and the low-energy gauge group is enhanced by an anomalous $\mathrm{U}(1)$ factor to $E_{7} \times \mathrm{U}(1)$ [17-19]. The bundle moduli (and matter fields) are charged under the enhanced $\mathrm{U}(1)$ symmetry denoted by

$$
C_{+}^{i} \in H^{1}\left(L^{2}\right), \quad C_{-}^{j} \in H^{1}\left(L^{\vee 2}\right),
$$

with the subscript \pm indicating the $\mathrm{U}(1)$ charge. We will take the extension class $\left\langle C_{+}^{i}\right\rangle \neq 0$ not far from the zero in $H^{1}\left(X, L^{2}\right)$ and keep $\left\langle C_{-}^{j}\right\rangle=0$ in the following.

The nontrivial F-term comes from the Gukov-Vafa-Witten superpotential [48, 49]. For bundle considered in (2.7), one can show that superpotential from the contribution of bundle moduli is [13]

$$
W=\lambda_{i j}\left(\mathfrak{z}_{0}\right) C_{+}^{i} C_{-}^{j}+\ldots
$$

where the coefficients $\lambda_{i j}$ depends on the complex structure moduli $\mathfrak{z}_{0}^{I} \in H^{1}(X, T X)$ explicitly. Note that for the generic complex structure, the holomorphic function $\lambda_{i j}\left(\mathfrak{z}_{0}\right) \neq 0$. As a result, the fields $C_{+}^{i}$ and $C_{-}^{j}$ are massive. However, on the special locus in complex structure moduli space, $\lambda_{i j}\left(\mathfrak{z}_{0}\right)=0$, which indicates that $C_{+}^{i}$ and $C_{-}^{j}$ are massless. The non-trivial VEVs of them define the non-Abelian $\mathrm{SU}(2)$ bundle [19]. This corresponds to the "jumping" of the Ext group described above.

The F-terms of the bundle moduli follows directly from the superpotential above. In particular, the F-terms of $C_{+}^{i}$ vanish while the other one becomes

$$
F_{C_{-}}=\frac{\partial W}{\partial C_{-}^{j}}=\lambda_{i j}\left(\mathfrak{z}_{0}\right)\left\langle C_{+}^{i}\right\rangle .
$$

As discussed above, since the initial complex structure is chosen in the special locus of the moduli space, hence, $\lambda_{i j}\left(\mathfrak{z}_{0}\right)=0$. Consider fluctuations of $\mathfrak{z}_{0}^{I} \rightarrow \mathfrak{z}_{0}^{I}+\delta \mathfrak{z}$. If these fluctuations are within the sub-locus where $H^{1}\left(X, L^{2}\right) \neq 0$ (denoted by $\mathfrak{z}_{\|}^{a}$ ), the new complex structure is still in the sub-locus and hence $\lambda_{i j}\left(\mathfrak{z}_{0}\right)=0$ and $F_{C_{-}}$vanishes. However, for the fluctuations away from this sub-locus (denoted by $\mathfrak{z}_{\perp}^{a}$ ), the new complex structure is a generic element in the moduli space and $\lambda_{i j}\left(\mathfrak{z}_{0}\right) \neq 0$. Up to the quadratic order, we have the non-zero F-term of $F_{C_{-}}$and the potential is given by

$$
V=\left|\frac{\partial \lambda_{i j}\left(\mathfrak{z}_{0}\right)}{\partial \mathfrak{z}_{\perp}^{I}}\left\langle C_{+}^{i}\right\rangle\right|^{2}\left|\delta_{\mathfrak{z}}^{I}\right|^{2}+\ldots
$$

This gives mass to the moduli $\mathfrak{z}_{\perp}^{a}$ and these degrees of freedom are stabilized. The number of these moduli is the same as the $\operatorname{Im}(\alpha)$ computed in the Atiyah sequence.

In summary, we review the complex structure moduli stabilization in Calabi-Yau compactification due to the presence of holomorphic bundles. The "true" moduli are those that keep the bundle holomorphic, while the other moduli are stabilized. With a simple rank two extension bundle (2.7), we demonstrate that $\operatorname{Ker}(\alpha)$ can be calculated by an analysis of 
"jumping" Ext group, which is equivalent to the direct computation from the sequence (2.4) and much easier computationally. Next, we will focus on the moduli stabilization on an elliptically fibered threefold and study how this mechanism works in that context.

\section{Complex structure moduli stabilization mechanisms}

An elliptically fibered Calabi-Yau threefold $X$ consists of a complex two-surface $B$ and an analytic map

$$
\pi: X \rightarrow B
$$

with the property that for a generic point $b \in B$, the fiber $E_{b}=\pi^{-1}(b)$ is an elliptic curve. In addition, we will require that there exist a global section $\sigma: B \rightarrow X$ that assigns to every point $b \in B$ the zero element $\sigma(b)=p \in E_{b}$ discussed below. Let $K_{B}$ be the canonical bundle of $B$. The Weierstrass form describes the elliptic fibration as

$$
y^{2}-x^{3}-f x z^{4}-g z^{6}=0,
$$

where $f$ and $g$ are sections of $K_{B}^{-4}$ and $K_{B}^{-6}$, and $(x, y, z)$ are global sections of $\mathcal{O}_{X}(2 \sigma) \otimes$ $K_{B}^{-2}, \mathcal{O}_{X}(3 \sigma) \otimes K_{B}^{-3}, \mathcal{O}_{X}(\sigma)$ respectively.

Consider a $\mathrm{SU}(n)$ vector bundle $V$ on $X$. The Chern character of $V$ can be written as the following general form,

$$
\operatorname{ch}(V)=n+\sigma \pi^{*} \eta+\omega[f]+\frac{1}{2} c_{3}(V),
$$

where $n$ is the rank of $V, \sigma$ is the section of the fibration, $\eta \in H^{2}(B, \mathbb{Z})$ is a divisor in the base, $\omega$ in an element in $H^{2,2}(B, \mathbb{Z})$, and $[f]$ is the fiber class of the elliptic fibration. The bundle $V$ can be constructed in terms of a spectral cover $\mathcal{S}_{n}$ and a spectral line bundle $\mathcal{N}[31,32]$. The spectral cover is a $n$ sheeted cover of $B$ given by a finite morphism $\pi_{S}: \mathcal{S}_{n} \rightarrow B$. In affine coordinates, where $z=1$, we can write it as

$$
\mathcal{S}_{n}=a_{0}+a_{2} x+a_{3} y+a_{4} x^{2}+a_{5} x^{2} y+\cdots+a_{n} x^{n / 2}=0
$$

Note that the last term is $a_{n} x^{(n-3) / 2} y$ when $n$ is odd. $\mathcal{N}$ is a line bundle over $\mathcal{S}_{n}$ (or a rank one coherent sheaf generally). The set of data $(\mathcal{S}, \mathcal{N})$ is called spectral data. It is shown $[31,32]$ that there is a one-to-one correspondence between holomorphic vector bundles and the spectral data,

$$
V \leftrightarrow\left(\mathcal{S}_{n}, \mathcal{N}\right)
$$

For an elliptically fibered Calabi-Yau threefold, the complex structure moduli space contains the moduli from the base $B$ and the moduli from the fibration. In fact, one can show that the moduli space $H^{1}(B, T B)$ is a subset of $H^{1}(X, T X)$. The detail of the derivation can be found in appendix A. The moduli space $H^{1}(B, T B)$ can be computed from the Euler sequence and adjunction formula. Suppose $B$ is defined as a hypersurface of an ambient space $A$, where $A$ can be the direct product of projective spaces $\mathbb{P}^{m_{1}} \times \ldots \times \mathbb{P}^{m_{n}}$ 
or in general toric varieties. The defining polynomial of $B$ is $p_{0} \in H^{0}(A, N)$ for the ample line bundle $N$. Then the tangent bundle is described by

$$
\begin{aligned}
0 & \left.\rightarrow O_{B}^{\oplus n} \stackrel{l_{1}}{\rightarrow} \bigoplus_{i=1, \ldots, n} O_{B}\left(D_{i}\right)^{\oplus\left(n_{i}+1\right)} \rightarrow T A\right|_{B} \rightarrow 0 \\
0 & \left.\rightarrow T B \rightarrow T A\right|_{B} \stackrel{l_{2}}{\rightarrow} N \rightarrow 0
\end{aligned}
$$

where $D_{i}$ are the restriction of the hyperplane divisors of each projective factor of the ambient space $A$, and the polynomial maps $l_{1}, l_{2}$ satisfy $l_{2} \circ l_{1}=p_{0}$. From the long exact sequence of cohomology groups, we have

$$
0 \rightarrow H^{0}(B, T B) \rightarrow H^{0}(B, T A) \stackrel{l_{2}}{\rightarrow} H^{0}(B, N) \rightarrow H^{1}(B, T B) \rightarrow H^{1}(B, T A) \stackrel{l_{2}}{\rightarrow} H^{1}(B, N) \rightarrow \ldots
$$

In general, it contains the polynomial deformations and the non-polynomial ones. We will only focus on the polynomial part $H_{\text {poly }}^{1}(B, T B) \subset H^{1}(B, T B)$ which is given by

$$
H_{\text {poly }}^{1}(B, T B)=\frac{H^{0}(B, N)}{\operatorname{Im}\left(l_{2}\right)} .
$$

The fibration $F$ of the Calabi-Yau threefold is taken to be in the Weierstrass form. The complex structure moduli space of $F$ can be determined following a similar calculation.

We wish to use the spectral data to find new examples of the complex structure moduli stabilization. There are three types of examples that we are going to consider. In the first type, some complex structure deformations are obstructed simply because the spectral cover cannot be defined globally everywhere in moduli space. In the second type, however, the Picard group of the base space jumps over some loci in the complex structure moduli, and if the spectral data depends explicitly on these new divisors, the deformations normal to this loci is obstructed. In the third type, the Picard group of the Calabi-Yau threefold jumps. More clearly, some specific algebraic curves inside the ambient four-fold will lie inside the Calabi-Yau threefold. For some specific bundles, these algebraic curves lie inside the spectral cover. ${ }^{5}$

\subsection{Type I: "jumping" extension group}

Consider the rank two extension bundle $V$ defined in (2.7). As discussed in section 2, on special loci in complex structure moduli space, cohomology $H^{2}\left(X, V \otimes V^{\vee}\right)$ jumps in dimension and the rank of Atiyah class $\alpha$ in (2.4) jumps as well. Hence, complex structure deformations perpendicular to this special loci will make $V$ non-holomorphic, and the associated moduli are stabilized [14]. In this subsection, we consider a similar set-up over an elliptically fibered Calabi-Yau threefold $\pi: X \rightarrow B$, and by analyzing such bundles using spectral cover/Fourier-Mukai construction, one gets a better insight about how exactly such bundles become non-holomorphic.

\footnotetext{
${ }^{5}$ Notation: from now on, we will denote the divisors in the base without writing the pullback $\pi^{*}$ on the front, e.g., we will write $\eta$ instead of $\pi^{*} \eta$. We also denote the spectral cover simply by $[\mathcal{S}]$ or $\mathcal{S}$ without emphasizing its degree.
} 
As in [33] we take $\mathcal{L}=\mathcal{O}_{X}\left(-\sigma+D_{b}\right)$. The extension group of $V$ is given by

$$
\begin{aligned}
\operatorname{Ext}_{X}^{1}\left(\mathcal{L}^{\vee}, \mathcal{L}\right) & =H^{1}\left(X, \mathcal{O}_{X}\left(-2 \sigma+2 D_{b}\right)\right) \\
& \simeq H^{0}\left(B, \mathcal{O}_{B}\left(2 D_{b}+K_{B}\right)\right) \oplus H^{0}\left(B, \mathcal{O}_{B}\left(2 D_{b}-K_{b}\right)\right)
\end{aligned}
$$

where the last isomorphism is derived by the Leray spectral sequence relative to the morphism $\pi$. It is also not hard to show [14] that the (holomorphic) deformations of $V$ i.e., $H^{1}\left(X, V^{\vee} \otimes V\right) \simeq H^{2}\left(X, V^{\vee} \otimes V\right)$ depend on the extension group above.

On the other hand, over the elliptically fibered Calabi-Yau $X$ the vector bundle $V$ can be parameterized by the spectral data $(\mathcal{S}, \mathcal{N})$ [31, 32], where $\mathcal{S}$ is a double cover of the base $B$ and $\mathcal{N}$ is a rank one coherent sheaf over $\mathcal{S}$. When $\mathcal{S}$ is smooth, $\mathcal{N}$ is simply a line bundle. The divisor class of $\mathcal{S}$ is determined by the topology of $V$,

$$
\begin{aligned}
{[\mathcal{S}] } & =2 \sigma+\eta, \\
\eta & =2 D_{b}+c_{1}(B) .
\end{aligned}
$$

Also the general form of the algebraic equation of $\mathcal{S}$ (when $X$ is given by the Weierstrass fibration) is given by,

$$
\begin{aligned}
\mathcal{S} & =a_{2} Z^{2}+a_{0} X, \\
a_{0} & \in H^{0}\left(B, \mathcal{O}_{B}\left(c_{1}(B)+2 D_{b}\right)\right), \quad a_{2} \in H^{0}\left(B, \mathcal{O}_{B}\left(-c_{1}(B)+2 D_{b}\right)\right) .
\end{aligned}
$$

By computing the Fourier-Mukai transformation of $V$, it is shown [33] that the polynomials $a_{2}$ and $a_{0}$ can be identified with the elements of the extension group (3.8). Therefore in the spectral cover language, the loci in the complex structure moduli where the group $H^{2}\left(X, V^{\vee} \otimes V\right)$ jumps correspond to the loci where it is possible to add new terms in the polynomials $a_{2}$ and $a_{0}$. Once one adds such terms in the spectral cover, the complex structure moduli are stabilized. The complex structure deformations that turn off such terms make the spectral cover non-algebraic, hence the associated vector bundle becomes non-holomorphic.

Example. To be more concrete, consider a Weierstrass elliptically fibered Calabi-Yau threefold $X$ over the following surface,

$$
B=\left[\begin{array}{l|l}
\mathbb{P}_{x}^{1} & 2 \\
\mathbb{P}_{y}^{2} & 2
\end{array}\right], \quad c_{1}(B)=H_{2} .
$$

Let us choose $D_{b}=-H_{1}+2 H_{2}$, where $H_{1}$ and $H_{2}$ are hyperplanes in $\mathbb{P}_{x}^{1}$ and $\mathbb{P}_{y}^{2}$ respectively. Therefore

$$
\begin{aligned}
& a_{0} \in H^{0}\left(B, \mathcal{O}_{B}(-2,5)\right), \\
& a_{2} \in H^{0}\left(B, \mathcal{O}_{B}(-2,3)\right) .
\end{aligned}
$$

Generic defining equation $P$ for $B$ can be written as

$$
P=x_{1}^{2} P_{1}(y)+x_{2}^{2} P_{2}(y)+x_{1} x_{2} P_{3}(y),
$$


where $P_{1}, P_{2}, P_{3}$ are degree two homogeneous polynomials in y. However, line bundles' cohomology over $B$ can jump over codimension one loci in complex structure moduli of the base $B$. In particular the cohomology of $\mathcal{O}_{B}(-2,3)$ and $\mathcal{O}_{B}(-2,5)$ can jump as, ${ }^{6}$

$$
\begin{aligned}
& h^{0}(B, O(-2,3))= \begin{cases}0, & P \text { generic } \\
3, & P=x_{1}^{2} P_{1}(y)+x_{2}^{2} P_{2}(y)\end{cases} \\
& h^{0}(B, O(-2,5))=\left\{\begin{array}{ll}
9, & P \text { generic } \\
13, & P=x_{1}^{2} P_{1}(y)+x_{2}^{2} P_{2}(y)
\end{array} .\right.
\end{aligned}
$$

The details of the computations are in the appendix C. This means the spectral cover, in general, can be written as,

$$
\mathcal{S}= \begin{cases}a_{0} Z^{2}, & P \text { generic } \\ a_{0} Z^{2}+a_{2} X, & P=x_{1}^{2} P_{1}(y)+x_{2}^{2} P_{2}(y),\end{cases}
$$

where in the second case $a_{0}$ is a rational polynomial [50] which is holomorphic over the hypersurface $P=0$,

$$
a_{2}=\frac{P_{1}(y)}{x_{2}^{2}} y_{1}, \frac{P_{1}(y)}{x_{2}^{2}} y_{2}, \frac{P_{1}(y)}{x_{2}^{2}} y_{3} .
$$

Similarly, the extra terms for the special defining equation $P=0$ is generally of form $\frac{P_{1}(y)}{x_{2}^{2}} f_{3}(y)$. Where $f_{3}$ is degree three polynomial in $y_{1}, y_{2}, y_{3}$, but we don't need the detailed form of it. Therefore in the complex structure locus where $P=x_{1}^{2} P_{1}(y)+x_{2}^{2} P_{2}(y)$ the extension group (3.8) jumps, and it is possible turn on these new terms. This corresponds to add the new terms in $a_{0}$ and $a_{2}$ in the spectral cover. We consider the new terms in $a_{0}$ and $a_{2}$ in turn.

First consider the $a_{2}$ term. When we turn on terms (3.19), the spectral cover is smooth. As it is shown in [31-33] generally the spectral line bundle $\mathcal{N}$ is

$$
\mathcal{N}=\mathcal{O}_{S}\left(\sigma+D_{b}\right)
$$

Therefore in general $\mathcal{N}$ depends on the divisor $\left.\sigma\right|_{S}$. The image of this curve on $B$ is the curve $\left(a_{2}=0\right)$. When one turns on the term $x_{1} x_{2} P_{3}(y)$ in (3.16), this curve "disappears". In other words, away form the locus (in the complex structure moduli space) where $P_{3}(y)$ in (3.16) is nonzero the divisor $-2 H_{1}+3 H_{2}$ is not effective anymore. This means after this complex structure deformation the curve $\left(a_{2}=0\right)$ mixes with $(2,0)$ or $(0,2)$ cycles. Therefore the corresponding curve $\left.\sigma\right|_{S}$ on the spectral cover is not algebraic anymore and mixes with $(2,0)$ or $(0,2)$ cycles. Hence the corresponding vector bundle becomes non-holomorphic.

Next, suppose $a_{2}=0$. Instead, we turn on the extra terms in $a_{0}$. In this case the spectral cover ${ }^{7} S=a_{0} Z^{2}$. Similar to the previous case if $a_{0}$ depends on the terms $\frac{P_{1}(y)}{x_{2}^{2}} f_{3}(y)$, the corresponding curve $a_{0}$ becomes non-algebraic after these complex structure deformations. Therefore the corresponding vector bundle $V$ becomes non-holomorphic.

\footnotetext{
${ }^{6}$ The surface $B$ is still smooth over this special complex structure locus.

${ }^{7}$ In this case the spectral cover is a union of two copies of the zero section $Z=0$ (which are "infinitesimally close" to each other) and the vertical surface $a_{2}=0$.
} 
The usual stabilization scenario of complex structure moduli by rank two vector bundles $[14,16]$ translates to whether the coefficients of the spectral cover equation exists globally. Therefore one may generalize this type of stabilization to vector bundles of arbitrary ranks simply by asking if a holomorphic vector bundle with the Chern character $\operatorname{ch}(V)=n+\sigma \eta+\omega[f]$ is given, then does the divisor $[S]=n \sigma+\eta$ have global section everywhere in the complex structure moduli?

Now, we will see how many complex structure moduli are stabilized in this example. If the initial defining polynomial is fixed by equation (C.7), the complex structure moduli space $H^{1}(B, T B)$ can be computed by the equation (3.6) and (3.7). It follows that the space of polynomial deformations $H_{\text {poly }}^{1}(B, T B)$ has dimension 6 with basis $\left\{d_{i}\right\}, i=1,2, \ldots, 6$. As discussed above, if any monomials in a polynomial deformation of $P$ contains $x_{0} x_{1}$ term and spectral cover depends on the extra terms in (3.17), then $\mathcal{S}$ will be non-algebraic after the deformation, and $V$ is not holomorphic any more. It turns out that there are 4 such deformations in $\left\{d_{1}, d_{2}, \ldots, d_{6}\right\} .8$ Therefore, we find that 4 out of 6 complex structure moduli of $B$ are stabilized.

\subsection{Type II: "jumping" Picard group}

For an elliptically fibered Calabi-Yau threefold, the Picard group of the base $B$ can jump in dimension, which can be used for moduli stabilization. The idea is that look for the loci in the complex structure moduli that the Picard group jumps (Noether-Lefschetz loci) and choose the spectral data to depend on the "new" elements of the Picard group. Therefore deforming the complex structure back is impossible because the corresponding vector bundle becomes non-holomorphic. We will explain this approach using an example.

Example. Consider the following surface

$$
B^{0}=\begin{array}{cccccc|c}
x_{1} & x_{2} & z_{1} & z_{2} & u_{1} & u_{2} & P \\
\hline 1 & 1 & 0 & 0 & 0 & 0 & 1 \\
0 & 1 & 1 & 1 & 0 & 0 & 3 \\
0 & 1 & 0 & 1 & 1 & 1 & 3
\end{array}
$$

where $\mathrm{P}$ is generically given by

$$
x_{1}\left(z_{1}^{3} f_{3}(u)+z_{1}^{2} z_{2} f_{2}(u)+z_{1} z_{2}^{2} f_{1}+z_{2}^{3} f_{0}\right)+x_{2}\left(z_{1}^{2} g_{2}(u)+z_{1} z_{2} g_{1}(u)+z_{2}^{2} g_{0}\right)=0 .
$$

Here, $f_{d}(u)$ and $g_{d}(u)$ represent generic degree $d$ homogeneous polynomials in $\left\{u_{1}, u_{2}\right\}$. This is actually the blow-up of $\mathbb{F}_{1}$ at the following six points

$$
z_{1}^{3} f_{3}(u)+z_{1}^{2} z_{2} f_{2}(u)+z_{1} z_{2}^{2} f_{1}+z_{2}^{3} f_{0}=z_{1}^{2} g_{2}(u)+z_{1} z_{2} g_{1}(u)+z_{2}^{2} g_{0}=0
$$

However the Picard number of $B$ is three $(\rho=3)$, but $h^{1,1}=8$. In other words, this surface is not favorable. This is because the six points are blown up "at the same time".

\footnotetext{
${ }^{8}$ Note that by an argument from linear algebra, this result does not change by choice of basis in $H_{\text {poly }}^{1}(B, T B)$.
} 
Note that the exceptional divisor can be identified by $-J_{1}+2 J_{2}+2 J_{3}$ is effective and has self-intersection $(-6)$. The global section of the corresponding line bundle is given as

$$
e=\frac{z_{1}^{2} g_{2}(u)+z_{1} z_{2} g_{1}(u)+z_{2}^{2} g_{0}}{x_{1}} .
$$

The zero locus $e=0$ corresponds to a union of six $\mathbb{P}^{1}$ 's each one is a $(-1)$ curve.

Let us tune the complex structure of the base $P$ to ${ }^{9}$

$$
x_{1}\left(z_{1}^{3} f_{3}(u)+z_{1}^{2} z_{2} f_{2}(u)+z_{1} z_{2}^{2} f_{1}+z_{2}^{3} f_{0}\right)+x_{2}\left(z_{1} h_{1}(u)+z_{2} h_{0}\right)\left(z_{1} l_{1}(u)+z_{2} l_{0}\right)=0 .
$$

Now there are two independent sets of three curves over

$$
z_{1}^{3} f_{3}(u)+z_{1}^{2} z_{2} f_{2}(u)+z_{1} z_{2}^{2} f_{1}+z_{2}^{3} f_{0}=z_{1} h_{1}(u)+z_{2} h_{0}=0,
$$

and over

$$
z_{1}^{3} f_{3}(u)+z_{1}^{2} z_{2} f_{2}(u)+z_{1} z_{2}^{2} f_{1}+z_{2}^{3} f_{0}=z_{1} l_{1}(u)+z_{2} l_{0}=0 .
$$

So the Picard number jumps to 4 . To make this more clear, one can rewrite the same geometry in the following way

$$
\begin{aligned}
& \begin{array}{cccccccc|cc|cc}
y_{1} & y_{2} & x_{1} & x_{2} & z_{1} & z_{2} & u_{1} & u_{2} & P_{1} & P_{2} \\
\hline 1 & 1 & 0 & 0 & 0 & 0 & 0 & 0 & 1 & 1
\end{array} \\
& B^{1}=\begin{array}{llllllll|ll}
0 & 0 & 1 & 1 & 0 & 0 & 0 & 0 & 1 & 0
\end{array} \text {. } \\
& \begin{array}{llllllllll}
0 & 2 & 0 & 1 & 1 & 1 & 0 & 0 & 2 & 3
\end{array} \\
& \begin{array}{llllllll|ll}
0 & 2 & 0 & 1 & 0 & 1 & 1 & 1 & 2 & 3
\end{array} \\
& P_{1}=y_{1} x_{2}\left(z_{1} h_{1}(u)+z_{2} h_{0}\right)+y_{2} x_{1}=0 \text {, } \\
& P_{2}=-y_{1}\left(z_{1}^{3} f_{3}(u)+z_{1}^{2} z_{2} f_{2}(u)+z_{1} z_{2}^{2} f_{1}+z_{2}^{3} f_{0}\right)+y_{2}\left(z_{1} l_{1}(u)+z_{2} l_{0}\right)=0 \text {. }
\end{aligned}
$$

The geometry above is equivalent to $(3.25)$ by eliminating $y_{1}$ and $y_{2}$. The two $(-1)$-curves and their global sections can be identified as

$$
\begin{array}{ll}
e_{1}=-J_{1}+J_{3}+J_{4}, & a_{1}=\frac{z_{1} l_{1}(u)+z_{2} l_{0}}{y_{1}}, \\
e_{2}=J_{1}-J_{2}+J_{3}+J_{4}, & a_{2}=\frac{y_{1}\left(z_{1} h_{1}(u)+z_{2} h_{0}\right)}{x_{1}} .
\end{array}
$$

Now, we want to use this jump in $\operatorname{Pic}(B)$ to stabilize the complex structure deformation that makes the divisor $e_{1}+e_{2}$ irreducible. To do this, consider, for example, a holomorphic vector bundle with Chern character,

$$
\operatorname{ch}(V)=n-\left(\sigma\left(\eta_{0}+e_{1}\right)+\omega[f]\right)+\ldots,
$$

where $\eta_{0}$ depends on $e_{1}+e_{2}$. Due to the special form of $\eta=\eta_{0}+e_{1}$, the spectral cover of $V$ is reducible. Therefore the spectral sheaf $\mathcal{N}$ is therefore defined as an extension,

$$
0 \rightarrow \mathcal{L}_{1} \rightarrow \mathcal{N} \rightarrow \mathcal{L}_{2} \rightarrow 0
$$

\footnotetext{
${ }^{9}$ The hypersurface is still smooth after this tuning.
} 
where $\mathcal{L}_{1}$ is a line bundle supported on $\pi^{*} e_{1}$, and $\mathcal{L}_{2}$ is a line bundle supported over smooth surface in the divisor class $n \sigma+\eta_{0}$. After a calculation similar to [33] one can show ${ }^{10}$

$$
c_{1}\left(\mathcal{L}_{2}\right)=\frac{1}{2}\left(c_{1}(B)-\left[\mathcal{S}_{0}\right]\right)+\lambda\left(n \sigma-\eta_{0}+n c_{1}(B)\right)+\lambda_{1} e_{1}
$$

The details on how to derive it is left in appendix B. What is important here is the dependence of the spectral sheaf on $e_{1}$. This means that deforming the complex structure back to the point where $e_{1}+e_{2}$ is irreducible is impossible for two reasons. First the spectral cover $[\mathcal{S}]=\left[\mathcal{S}_{0}\right]+e_{1}$ becomes non-effective, i.e. one cannot define the spectral cover globally. Second, if $\lambda_{1} \neq 0$ the line bundle $\mathcal{L}_{2}$ over $\mathcal{S}_{0}$ becomes non-holomorphic as we deform the complex structure.

We will see how the complex structure moduli of $B^{0}$ are stabilized in this example. Set the initial defining polynomial $P^{(0)}$ to be the special polynomial defined in (3.25). To simply the notation, let's write it as $P^{(0)}=x_{1} F_{1}^{(0)}+x_{2} F_{2}^{(0)}$ where $F_{1}^{(0)}=z_{1}^{3} f_{3}(u)+$ $z_{1}^{2} z_{2} f_{2}(u)+z_{1} z_{2}^{2} f_{1}+z_{2}^{3} f_{0}$ and $F_{2}^{(0)}$ is a reducible polynomial given by

$$
F_{2}^{(0)}=\left(A_{1} u_{1} z_{1}+A_{2} u_{2} z_{1}+A_{3} z_{2}\right)\left(B_{1} u_{1} z_{1}+B_{2} u_{2} z_{1}+B_{3} z_{2}\right),
$$

with $A_{i}$ 's and $B_{i}$ 's the coefficients. As discussed above, the base $B^{0}$ admits two algebraic divisors $e_{1}$ and $e_{2}$ given by equation (3.29). With them, one can define a holomorphic bundle $V$.

Now, consider the polynomial deformations of $P^{(0)}$ given by $\delta P=x_{1} \delta F_{1}+x_{2} \delta F_{2}$. At first, we keep our discussion in general and allow $\delta P$ to be any elements in $H^{0}\left(B^{0}, N\right)$. Eventually, we will restrict them to be the deformations in $H_{\text {poly }}^{1}\left(B^{0}, T B^{0}\right)$. To keep $V$ holomorphic, the term $F_{2}^{(0)}+\delta F_{2}$ in deformed defining polynomial $P^{(0)}+\delta P$ should remain reducible. Deformations satisfying this condition are called reducible deformations. Before studying complex structure moduli stabilization, one needs to find all the reducible deformations.

Obviously, $\delta P$ with $\delta F_{2}=0$ are reducible deformations because they have no effect to $F_{2}^{(0)}$. Let $M_{r}^{1}$ be the space of all such deformations. For deformations with $\delta F_{2} \neq 0$, it is not clear if $F_{2}^{(0)}+\delta F_{2}$ remain reducible. However, we know that the deformed $F_{2}$ term, if reducible, should be able to be written in the form of (3.34). The first-order deformation $\delta F_{2}$ just shifts the initial coefficients in (3.34) to a set of new coefficients $A_{i}+\delta A_{i}$ and $B_{i}+\delta B_{i}$. We will see this in details in appendix D. Notice that if $\delta F_{2}$ is a deformations that keeps of $F_{2}^{(0)}+\delta F_{2}$ reducible, then $\delta P=x_{2} \delta F_{2}$ is a reducible deformation of $P^{(0)}$.

To obtain the reducible deformations of $F_{2}^{(0)}$ it is sufficient to calculate the $\delta F_{2}$ obtained by a shift of each coefficient. For example, the reducible deformation corresponding to a shift of the first coefficient $A_{1}+\delta A_{1}$ is obtained by

$$
\begin{aligned}
F_{2} & =\left(\left(A_{1}+\delta A_{1}\right) u_{1} z_{1}+A_{2} u_{2} z_{1}+A_{3} z_{2}\right)\left(B_{1} u_{1} z_{1}+B_{2} u_{2} z_{1}+B_{3} z_{2}\right) \\
& =F_{2}^{(0)}+\delta A_{1} u_{1} z_{1}\left(B_{1} u_{1} z_{1}+B_{2} u_{2} z_{1}+B_{3} z_{2}\right) \\
& =F_{2}^{(0)}+\delta A_{1} e_{1}^{A} .
\end{aligned}
$$

\footnotetext{
${ }^{10}$ The term $\lambda_{1} e_{1}$ in $c_{1}\left(\mathcal{L}_{2}\right)$ have to be compensated with a term $-\lambda_{1} \mathcal{S}_{0}$ in $c_{1}\left(\mathcal{L}_{1}\right)$. They contribute to $c_{3}(V)$ and $\omega$, but since these are not important for the main purpose of this paper, we ignore further details.
} 
In a similar manner, one can obtain the reducible deformations from shifting the other coefficients

$$
\begin{array}{ll}
e_{1}^{A}=u_{1} z_{1}\left(B_{1} u_{1} z_{1}+B_{2} u_{2} z_{1}+B_{3} z_{2}\right), & e_{1}^{B}=u_{1} z_{1}\left(A_{1} u_{1} z_{1}+A_{2} u_{2} z_{1}+A_{3} z_{2}\right) \\
e_{2}^{A}=u_{2} z_{1}\left(B_{1} u_{1} z_{1}+B_{2} u_{2} z_{1}+B_{3} z_{2}\right), & e_{2}^{B}=u_{2} z_{1}\left(A_{1} u_{1} z_{1}+A_{2} u_{2} z_{1}+A_{3} z_{2}\right) \\
e_{3}^{A}=z_{2}\left(B_{1} u_{1} z_{1}+B_{2} u_{2} z_{1}+B_{3} z_{2}\right), & e_{3}^{B}=z_{2}\left(A_{1} u_{1} z_{1}+A_{2} u_{2} z_{1}+A_{3} z_{2}\right) .
\end{array}
$$

The linear combinations of $\left\{e_{i}^{A}\right\}$ and $\left\{e_{j}^{B}\right\}$ give reducible deformations of $\delta F_{2}$. However, there is a relation among them

$$
F_{2}^{(0)}+A_{1} e_{1}^{A}+A_{2} e_{2}^{A}+A_{3} e_{3}^{A}=F_{2}^{(0)}+B_{1} e_{1}^{B}+B_{2} e_{2}^{B}+B_{3} e_{3}^{B}
$$

as one can easily check with the equation (3.34) and (3.35). Thus, only 5 of $\left\{e_{i}^{A}\right\}$ and $\left\{e_{j}^{B}\right\}$ are independent and, when multiplying them by $x_{2}$, they span a vector space denoted by $M_{r}^{2}$ containing all the reducible deformations with $\delta F_{2} \neq 0$. Therefore, the space of all reducible deformations for $P^{(0)}$ is $M_{r}=M_{r}^{1} \cup M_{r}^{2}$.

The complex structure moduli space $H^{1}\left(B^{0}, T B^{0}\right)$ is determined by the equation (3.6) and (3.7). It follows that the space of polynomial deformations $H_{\text {poly }}^{1}\left(B^{0}, T B^{0}\right)$ has dimension $5,{ }^{11}$ spanned by homogeneous polynomials $d_{i}, i=1,2, \ldots, 5$. The intersection $H_{\text {poly }}^{1}\left(B^{0}, T B^{0}\right) \cap M_{r}$ contains all the polynomial deformations from $B^{0}$ that keep $V$ holomorphic and contribute to the nontrivial kernel of Atiyah class in (2.4). The dimension of this intersection determines the number of the "true" moduli in the effective theory while the moduli in the complement of the intersection are stabilized. In our example, $M_{r} \cap$ $H_{\text {poly }}^{1}\left(B^{0}, T B^{0}\right)$ is a 4 dimensional space. Thus, 1 complex structure moduli is stabilized. ${ }^{12}$

\subsection{Type III: emergent algebraic cycle}

In the final example, we find that there exist algebraic curves in an elliptically fibered Calabi-Yau threefold for special complex structure of the fibration. As in the previous example, defining a vector bundle with these curves stabilizes the complex structure moduli in the fibration.

Consider an elliptic fibered Calabi-Yau threefold $\pi: X \rightarrow B$ with Weierstrass fibration given by

$$
F_{W}^{(0)}=y^{2}-x^{3}-f x z^{4}-g z^{6}=0, \quad g=\alpha g^{\prime},
$$

where $f \in H^{0}\left(B, K_{B}^{-4}\right)$ and $g \in H^{0}\left(B, K_{B}^{-6}\right)$ chosen to be a product of two polynomials $\alpha$ and $g^{\prime}$ with correct degree. This reducible polynomial $g$ allow us to define an algebraic 2-cycle on $X$

$$
[C]=\{x=y=\alpha=0\} .
$$

\footnotetext{
${ }^{11}$ There are also non-polynomials deformations, but we will not consider them here.

${ }^{12}$ One could guess this result by comparing the complex structure of $B^{0}$ and $B^{1}$. Since the complex structure of $B^{1}$ is "frozen" in the sense that it is impossible to "glue" the divisors $e_{1}$ and $e_{2}$ into a single divisor $e$, but any other complex structure deformation of $B^{0}$ is in a one-one correspondence with the complex structure deformations of $B^{1}$. So the space $H^{1}\left(T B^{1}\right)$ corresponds to the flat (unstabilized) directions. Finally one can check that $h_{\text {poly }}^{1}\left(B^{1}, T B^{1}\right)-h_{\text {poly }}^{1}\left(B^{0}, T B^{0}\right)=-1$ as expected.
} 
Note that this algebraic cycle is independent of the intersection of divisors $\sigma \cdot \pi^{*} D_{b}$ and the fiber class $[f]$. Now consider the spectral cover of a general rank $n$ vector bundle $V_{n}$

$$
\mathcal{S}\left(V_{n}\right)=a_{0} z^{n}+a_{2} z^{n-2} x+\cdots+a_{n} x^{n / 2} .
$$

The 2-cycle $[C]$ intersect with spectral cover at a finite number of points. However, after choosing $a_{0}$ to be reducible

$$
a_{0}=\alpha a_{0}
$$

the curve $[C]$ can lie inside the spectral cover. So if the spectral sheaf $\mathcal{N}$ depends on this new divisor inside $\mathcal{S}\left(V_{n}\right)$ we can stabilize the complex structure moduli, simply because any deformation that removes $[C]$ from $\mathcal{S}\left(V_{n}\right)$ makes $\mathcal{N}$ and hence the bundle $V_{n}$ non-holomorphic. However, a Fourier-Mukai analysis as in [33] shows that if the Chern classes of the vector bundle do not depend on the 2-cycle $[C]$, then the spectral line bundle also does not depend on the new divisor $[C]$ in $\mathcal{S}\left(V_{n}\right)$. Therefore the complex structure moduli cannot be stabilized. So suppose the Chern character of $V_{n}$ is of the form (See appendix B for details)

$$
C h\left(V_{n}\right)=n-(\sigma \cdot \eta+\omega[f]+[C])+\frac{1}{2} c_{3}\left(V_{n}\right)
$$

By doing similar calculations as in [33] we can derive the topological constraints on the divisor class of $S\left(V_{n}\right)$, Chern classes of $V_{n}$ and $\mathcal{L}$. We will not repeat them here. However, we just mention the part of the results that we will need, ${ }^{13}$

$$
\begin{aligned}
{\left[\mathcal{S}\left(V_{n}\right)\right] } & =n \sigma+\eta+\pi_{*}[C], \\
c_{1}(\mathcal{N}) & =\frac{1}{2}\left(\left[S\left(V_{n}\right)\right]-c_{1}(B)\right)+\lambda\left(n \sigma-\eta+n c_{1}(B)\right)+\left(\frac{1}{n} \pi_{*}[C]-[C]\right) .
\end{aligned}
$$

Therefore $\mathcal{N}$ explicitly depends on the divisor $[C]$ in the spectral cover. This dependence (partially) stabilize both complex structure and vector bundle moduli.

Consider a deformation of Weierstrass fibration $F_{W}^{(0)} \rightarrow F_{W}^{(0)}+\delta g z^{6}$, which induces a deformation to function $g$ by

$$
\alpha g^{\prime} \longrightarrow g
$$

If the deformed $g$ can be expressed as a product of two polynomials (having the same degree with $\alpha$ and $g^{\prime}$ ), then the algebraic curve $[C]$ defined in (3.38) still exist, and the bundle $V$ remain holomorphic. However, if the function $g$ after deformation is irreducible, the curve $[C]$ is not algebraic anymore, and the vector bundle defined by the spectral line bundle (3.43) which explicitly depends on $[C]$ becomes non-holomorphic. Therefore the associated complex structure moduli of $F_{W}$ can be stabilized. As we will explain in section 4, these polynomial deformations map to complex structure moduli in the F-theory dual picture, and a four-flux stabilizes them.

\footnotetext{
${ }^{13}$ Note that we should choose $\alpha$ such that $\frac{1}{n} \pi_{*}[C]$ be integral.
} 
Example. Let $\pi: X \rightarrow \mathbb{P}^{2}$ be an elliptically fibered Calabi-Yau threefold with base $\mathbb{P}^{2}$. The fibration is Weierstrass introduced in equation (3.2) with $f \in H^{0}\left(\mathbb{P}^{2}, O(12)\right)$ and $g \in H^{0}\left(\mathbb{P}^{2}, O(18)\right)$. Let $\left\{u_{1}, u_{2}, u_{3}\right\}$ be the homogeneous coordinates of $\mathbb{P}^{2}$. Choose the initial fibration to be

$$
f^{(0)}=F_{12}(u), \quad g^{(0)}=\alpha F_{16}(u)
$$

where $F_{12}$ and $F_{16}$ are generic polynomials with degrees 12 and 16 and $\alpha$ is a degree 2 homogeneous polynomial. As shown in (3.38), the fibration with reducible $g$ admits an algebraic curve $[C]$ in $\mathbb{P}^{2}$, which will be used to define the topology of vector bundles.

Consider a $\mathrm{SU}(2)$ extension bundle $V$ over $X$. The spectral cover construction of $V$ introduced in equation (3.11) is $S=a_{2} z^{2}+a_{0} x$ where $x, z$ are coordinates of the fibration. If $D_{b}=O(2)$, then $a_{2}$ and $a_{0}$ should be global sections of $O_{B}(7)$ and $O_{B}(1)$. Let's take them to be

$$
a_{0}=F_{1}\left(u_{1}, u_{2}, u_{3}\right), \quad a_{2}=\alpha F_{5}\left(u_{1}, u_{2}, u_{3}\right)
$$

where $F_{d}$ is a generic homogeneous polynomial with degree $d$. As discussed in (3.41) and (3.43), if the Chern character of $V$ depends on the algebraic 2-cycle $[C]$, then the Chern class of spectral sheaf depends on $[C]$ explicitly. Thus, the holomorphy of $V$ depends on the existence of the algebraic cycle $[C]$ that will be used to stabilize complex structure moduli in a second.

We will study how many complex structure moduli can be stabilized in this example. The elliptically fibered Calabi-Yau manifold $X$ can be regarded as

$$
X=\begin{array}{cccccc|c}
u_{1} & u_{2} & u_{3} & x & y & z & P \\
\hline 1 & 1 & 1 & 6 & 9 & 0 & 18 \\
0 & 0 & 0 & 2 & 3 & 1 & 6
\end{array}
$$

With it, one can compute the complex structure moduli space $H^{1}(X, T X)$ from the equation (3.6) and (3.7). Again, we will only focus on the polynomial deformations $H_{\text {poly }}^{1}(X, T X)$ which has dimension 272 . Since the base $\mathbb{P}^{2}$ is rigid, all the complex structure moduli are coming from the fibration, i.e. deformations of $f$ and $g .{ }^{14}$

As discussed above, deformations that keep $V$ holomorphic are those preserve the reducibility of $g=\alpha F_{16}(u)$. Again, we will call them reducible deformations. Obviously, any polynomial without $z^{6}$ terms is reducible deformation. While, the reducible deformations with $z^{6}$ terms can be calculated from the method in appendix D. We find that these deformations can be generated from 159 polynomial deformations $e_{i}^{A}, i=1,2, \ldots, 6$ and $e_{j}^{B}, i=1,2, \ldots, 153$, which are obtained by deforming the coefficients in $\alpha$ and $F_{16}(u)$ respectively. In fact, the space of these deformations denoted by $M_{r}^{2}$ has dimension 158 because there is a nontrivial relation in (D.8) between $\left\{e_{i}^{A}\right\}$ 's and $\left\{e_{j}^{A}\right\}$ 's.

Finally, we will see how many polynomial deformations $H_{\text {poly }}^{1}(X, T X)$ are stabilized. One needs to split 272 deformations found earlier into two groups. One group is proportional to $z^{6}$, which have no effect on $g$ and should be considered as the moduli of the effective

\footnotetext{
${ }^{14}$ Indeed, the degree of freedom of $f$ and $g$ is $h^{0}\left(\mathbb{P}^{2}, O(12)\right)+h^{0}\left(\mathbb{P}^{2}, O(18)\right)=91+190=281$. Considering the coordinates redefinition of $\mathbb{P}^{2}$, one find the independent degree of freedom of $f$ and $g$ is 272 , which matches the number of complex structure moduli counted in $h^{1}(X, T X)=272$.
} 
theory. There are another 82 of them. These ones contain terms proportional to $z^{6}$ and could have an effect on the reducibility of $g$. It turns out that there are 190 such polynomials. Their coefficients of $z^{6}$ is denoted by $d_{i}, i=1,2, \ldots, 190$. Then, one needs to study how many of them, or their linear combinations can give rise to reducibility preserving deformations in $M_{r}^{2}$. Direct computations shows that all 158 polynomials in $M_{r}^{2}$ can be expressed in the complex structure moduli $\left\{d_{i}\right\}$. Thus, there are 241 polynomial deformations that preserves the reducible of $g$ while there are 32 complex structure moduli are stabilized.

\section{Discussion and comments on F-theory dual}

In this section, we briefly describe the F-theory translation of the Heterotic examples mentioned before. When the spectral cover is smooth, the duality procedure is well-known [51]. The geometry of the F-theory half-Calabi-Yau fourfold (more precisely $d P_{8}$ fibration) is given by (for a $\mathrm{SU}(n)$ bundle with $n \leq 5$ ),

$$
Y^{2}=X^{3}+f X v^{4}+g v^{6}+u v^{5-n} S_{n}(X, Y, v),
$$

where the term in the parenthesis is the spectral cover of a $\mathrm{SU}(n)$ vector bundle (with $z$ is replaced by $v)$, and $(X, Y, u, v)$ are the homogeneous coordinates of $\mathbb{W} \mathbb{P}^{3211}$. In addition, using the cylinder map, one constructs the four-flux. The details can be found in [51]. It is only sufficient to mention that the part of the $c_{1}(\mathcal{L})$ that lies in the kernel of $\pi_{S}: S \rightarrow B$, lifts to the four-flux in F-theory language,

$$
\left.\begin{array}{c}
c_{1}(\mathcal{L})=\frac{1}{2}\left(c_{1}(B)-[S]\right)+\gamma \\
\pi_{S *} \gamma=0
\end{array}\right\} \Rightarrow \gamma \longrightarrow G_{4} \sim P_{C y l}^{*} \gamma
$$

where $P_{C y l}$ is the cylinder map which is a $\mathbb{P}^{1}$ fibration over the spectral cover $S_{n}$,

$$
P_{C y l}:\left\{S_{n}(X, Y, v)=0 \subset X_{F}\right\} \stackrel{\mathbb{P}^{1}}{\rightarrow} S_{n}(x, y, z) \subset X_{H e t} .
$$

In terms of the local 7-brane data, there is a doublet $(E, \Phi)$, called Higgs bundles, which consists of a holomorphic bundle $E$ on $B$ (where 7-brane wraps) and a "Higgs" field $\Phi \in$ $H^{0}\left(E \otimes E^{*} \otimes K(\eta)\right)$ which satisfy the Hitchin system equations [52]. One can study the Higgs bundles by spectral data,

$$
(E, \Phi) \leftrightarrow\left(S_{H}, \mathcal{L}_{H}\right)
$$

where $S_{H}$ is a finite cover of $B$ defined as a subvariety of $\operatorname{Tot}(K)$,

$$
S_{H}=\operatorname{Det}(\lambda 1-\Phi)=0,
$$

where $\lambda$ is the tautological section of $\operatorname{Tot}(K)$, and $\mathcal{L}_{H}$ is a line bundle over $S_{H}$. The Higgs bundle $(E, \Phi)$ can be reconstructed by the spectral data as,

$$
\begin{aligned}
E & =\pi_{*} \mathcal{L}_{H}, \quad \Phi=\pi_{*} \lambda, \\
\pi_{S}: S_{H} & \rightarrow B .
\end{aligned}
$$


The Higgs bundle spectral data is very similar to the Heterotic spectral data derived from the Fourier transform of $V$. They are indeed related to each other by cylinder construction [53].

Finally, note that what is responsible for the complex structure is the F-term derived from the Gukov-Vafa-Witten like superpotentials, which takes the following forms in Heterotic, F-theory, and 7-brane

$$
\begin{array}{ccccc}
\text { Heterotic } & \leftrightarrow & \text { F-theory } & \leftrightarrow & 7 \text {-brane } \\
W=\int_{X_{H e t}} \omega_{3} \wedge \Omega_{3} & \leftrightarrow & W=\int_{X_{F}} G_{4} \wedge \Omega_{4} & \leftrightarrow & W=\int_{B} \operatorname{Tr}\left(F_{2} \wedge \Phi\right)
\end{array}
$$

Where $\Omega$ corresponds to the complex structure top-form and $F_{2}$ is the curvature twoform of $E$. With this introduction, we move forward to briefly explain the "image" of the moduli stabilization scenarios of the Heterotic string in the dual F-theory. Locally over the 7-brane, the story is similar to the Heterotic version. The deforming away from special loci in complex structure moduli of the base makes the Higgs bundle non-holomorphic. The term $F_{2} \wedge \Phi$ in the superpotential makes some components of $\Phi$ massive. Generally, since the Higgs field corresponds to the fluctuations of the 7-brane in the normal directions, this means that away from some special loci in the complex structure moduli of $B$, the 7-brane cannot "wiggle" in specific ways. This can be seen more clearly, by noting that the spectral cover's coefficients can be identified with the Casimir's of the Higgs field.

The above arguments were local, but in the following, we will mainly focus on the effect of these deformations on the $G_{4}$ flux. The type III example can only be explained in terms of the global $G_{4}$ flux stabilization rather than local two-flux.

Type I. We already know in the extension class of $V$ in (2.7) appeared as the new terms in $a_{0}$ and $a_{2}$ in (3.8). By the well-known arguments briefly reviewed above, these jumps in $a_{0}$ and $a_{2}$ correspond to new jumps in Casimir's of $\Phi$, in other words, new zero modes in fluctuations of the 7-brane.

New zero modes in the vector bundle's moduli space in Heterotic string corresponds to new zero modes in deformations of the 7-brane in F-theory.

To construct the $G_{4}$ flux, assume the spectral cover is smooth,

$$
S=a_{2} x+a_{0} z^{2} .
$$

Then by the introduction above, $G_{4}$ flux is related to the divisor $\gamma$ in $c_{1}(\mathcal{N})$. But $\gamma=0$ for SU(2) bundles. ${ }^{15}$ So one may naively expect that the $G_{4}$ flux must vanish in this case. But, note that in this case, there are a finite number of "vertical components" (i.e., isolated elliptic curves) inside the spectral cover located above the points $a_{0}=a_{2}=0$ in the base $B$. In the dual F-theory geometry, such vertical components become 4 -cycles $C_{4}$ by cylinder map, which are (at least topologically) elliptic curves times a $\mathbb{P}^{1}$.

One can use these cycles to construct $G_{4}$ flux,

$$
G_{4} \longrightarrow C_{4}+\text { Corrections, }
$$

\footnotetext{
${ }^{15} \gamma$ induces non-zero chirality in the effective theory, but for $\mathrm{SU}(2)$ bundles, the chirality must be zero. The vanishing of $\gamma$ can also be seen from direct spectral cover calculations.
} 
where the corrections are terms we need to add such that

$$
G_{4} \cdot \sigma \cdot \pi^{*} D_{\text {base }}=0
$$

Note that this $G_{4}$ flux only exists when the terms $a_{2}$ and $a_{0}$ in the spectral cover are not zero. Therefore, when we move to a generic point in the complex structure moduli where there are no jumps in $a_{2}$ and $a_{0}$, the $G_{4}$ flux constructed above "disappears" i.e., $G_{4}$ becomes non-holomorphic, which by the Gukov-Vafa-Witten superpotential makes the corresponding complex structure deformation massive. ${ }^{16}$

Type II. In this case, the spectral cover is smooth, and as explained in subsection 3.2, after the complex structure deformation, the spectral cover is not algebraic anymore. So an argument similar to the one mentioned above is (when $a_{2} \neq 0$ ) also applies in this case. In other words, the dual Higgs bundle spectral data on the F-theory side is also required to be holomorphic, and the F-term equations obstruct these deformations in the 7-brane worldvolume theory.

In addition, $\gamma$ term in (4.2) can be non-zero in general (for $n>2$ ). The general form of $\gamma$ in $[31,32]$ is

$$
\gamma=\left.\lambda\left(n \sigma-\eta+n c_{1}(B)\right)\right|_{S} .
$$

The curves (divisors) $\left.\sigma\right|_{S}$ and $\eta_{S}$, and hence $\gamma$ and $G_{4}$ in (4.2), are holomorphic as long as the spectral cover is holomorphic. Therefore, if the spectral cover's coefficients depend on the new terms that appear in the special loci of the complex structure moduli space, then it is impossible to move away from those points since $\gamma$. Hence, the corresponding $G_{4}$ flux becomes non-holomorphic.

In the example we considered here, $\gamma$ depends on the new divisor $e_{1}$,

$$
\gamma=\gamma_{0}+\lambda_{1} e_{1}
$$

Of course $\pi_{s *} \gamma \neq 0$ now, so one should modify the corresponding four-flux, but the point is that this four-flux is only holomorphic on the jumping locus.

Type III. In this type the $\gamma$ is modified as ${ }^{17}$

$$
\gamma=\lambda\left(n \sigma-\eta+n c_{1}(B)\right)+\left(\frac{1}{n} \pi_{*}[C]-[C]\right) .
$$

As mentioned before, on the Heterotic side, the dependence of (3.43) on $[C]$ stabilize both the complex structure moduli and the vector bundle moduli. But in F-theory dual, both (3.37) and (3.40) corresponds to complex structure deformation on the F-theory side.

\footnotetext{
${ }^{16}$ The Calabi-Yau four-fold of F-theory has $(4,6,12)$ singularity over the points corresponding to the vertical components of $S$. Naively one may intend to blow these singularities up, but this is only possible when the Heterotic "bundle" (coherent sheaf) is a direct sum of a vector bundle and small instantons localized on the elliptic curves.

${ }^{17}$ Note that $\pi_{S *}\left(\frac{1}{n} \pi_{*}[C]-[C]\right)=0$.
} 
The corresponding (horizontal) four-flux that is responsible for stabilizing the F-theory complex structure is already studied [35]. A quick look at the defining relation for the Ftheory geometry (4.1) makes it clear that with this particular constraint over the geometry of the Heterotic Calabi-Yau and spectral cover, there is an algebraic four-cycle,

$$
C_{4}:=\{X=Y=\alpha=0\} \subset X_{F},
$$

which can be used to construct an "algebraic four-flux". It is also clear that $C_{4}$ is a $\mathbb{P}^{1}$ fibration (cylinder) over the algebraic curve $[C]$ in the Heterotic geometry. Therefore we can identify $C_{4}$ with the $P_{C y l}^{*}[C]$ part of the four-flux induced by the spectral cover.

Finally, note that contrary to type I and II, the two-flux localized inside the 7-brane is not responsible for stabilizing the complex structure moduli. This is because the vector bundle in the Heterotic side is identified with the spectral data living in the compact elliptic fibration over $B$. In contrast, the Higgs bundle on the 7-brane is identified with spectral data that live in the non-compact fibration $\operatorname{Tot}\left(K_{B}\right)$. Therefore in local F-theory/Heterotic duality, only the part of Heterotic spectral data in the patch that contains the zero section is identified with the Higgs bundle spectral data. In other words,

$$
\left.\left(\mathcal{S}_{\text {Het }}, \mathcal{N}_{\text {Het }}\right)\right|_{\sigma \text {-patch }} \longleftrightarrow\left(\mathcal{S}_{\text {Higgs }}, \mathcal{N}_{\text {Higgs }}\right)
$$

However, remember that

$$
\sigma \cdot[C]=0
$$

\section{Conclusion}

In this paper, we have studied how holomorphic bundles can stabilize complex structure moduli of an elliptically fibered Calabi-Yau threefold in the context of a heterotic compactification. In general, these moduli can come from the complex structure moduli of the base and the complex structure moduli of the fibration. For vector bundles defined in terms of spectral data, we find three approaches that both of these moduli can be stabilized with algebraic cycles existing only on special loci in the moduli space. We develop practical tools to identify the stabilized moduli and present concrete examples for all three approaches. As a result, we show that complex structure moduli of the elliptically fibered Calabi-Yau threefold can be effectively stabilized by the holomorphic bundles.

As mentioned in the introduction, one of the advantages of working with spectral covers is the manifest connection with the F-theory and hence type II models. In other words, the complex structure moduli stabilization scenarios are already well known, at least conceptually, in both type II and Heterotic string, but apparently, they look very different from each other. But both of these theories can be seen as different limits of Ftheory. By describing the moduli stabilization in terms of spectral data, we could see the corresponding stabilization scenario in the dual F/type II theory. In particular, we have shown that in the type I/II examples, the dual stabilization is made possible by the flux inside the 7-brane worldvolume. In contrast, in the type III example, the dual F-theory geometry is stabilized by a horizontal algebraic $G_{4}$ flux. 


\section{Acknowledgments}

W. Cui would like to thank James Gray for the useful discussion. This work was supported by IBS under the project code, IBS-R018-D1.

\section{A Complex structure moduli space of elliptically fibered Calabi-Yau threefold}

In this appendix, we briefly discuss the relation between the complex structure moduli space of an elliptically fibered Calabi-Yau threefold $H^{1}(X, T X)$ and the complex structure moduli space of its base $H^{1}(B, T B)$. We assume $B$ is a Fano surface $\left(K_{B}^{-1}\right.$ is ample) and $h^{1}\left(\mathcal{O}_{B}\right)=0$.

One can use the adjunction sequence for the section, $\sigma$, of the fibration to relate the tangent bundle of the base $T B$ with the tangent bundle of the Calabi-Yau manifold $X$,

$$
\left.0 \rightarrow T B \rightarrow T X\right|_{B} \rightarrow \mathcal{N}_{B / X}=K_{B} \rightarrow 0 .
$$

From the sequence above, one can see, as long as the base $B$ is simply connected, $H^{1}(T B) \simeq$ $H^{1}\left(B,\left.T X\right|_{B}\right)$. So we should find the relation between $\left.T X\right|_{B}$ and $T X$,

$$
\left.0 \rightarrow T X \otimes \mathcal{O}_{X}(-\sigma) \rightarrow T X \rightarrow T X\right|_{B} \rightarrow 0 .
$$

The corresponding long exact sequence is,

$$
\begin{aligned}
0 & \rightarrow H^{0}\left(T X \otimes \mathcal{O}_{X}(-\sigma)\right) \rightarrow H^{0}(T X) \rightarrow H^{0}\left(\left.T X\right|_{B}\right) \rightarrow \\
& \rightarrow H^{1}\left(T X \otimes \mathcal{O}_{X}(-\sigma)\right) \rightarrow H^{1}(T X) \rightarrow H^{1}\left(\left.T X\right|_{B}\right) \rightarrow \\
& \rightarrow H^{2}\left(T X \otimes \mathcal{O}_{X}(-\sigma)\right) \rightarrow H^{2}(T X) \rightarrow H^{2}\left(\left.T X\right|_{B}\right) \rightarrow \\
& \rightarrow H^{3}\left(T X \otimes \mathcal{O}_{X}(-\sigma)\right) \rightarrow H^{3}(T X) \rightarrow 0 .
\end{aligned}
$$

We will show bellow that as long as $K_{B}^{-1}$ is ample, the homeomorphism $H^{1}\left(X,\left.T X\right|_{B}\right) \simeq$ $H^{1}(B, T B) \rightarrow H^{2}\left(T X \otimes \mathcal{O}_{X}(-\sigma)\right)$ have to be zero. To see this, we will use the following sequences,

$$
\begin{aligned}
& \left.\left.0 \rightarrow T X \otimes \mathcal{O}_{X}(-\sigma) \rightarrow T \mathcal{A}\right|_{X} \otimes \mathcal{O}_{X}(-\sigma) \rightarrow \mathcal{N}\right|_{X} \otimes \mathcal{O}_{X}(-\sigma) \rightarrow 0, \\
& \left.\left.0 \rightarrow T_{\bar{\pi}}\right|_{X} \rightarrow T \mathcal{A}\right|_{X} \rightarrow \pi^{*} T B \rightarrow 0, \\
& \left.0 \rightarrow \mathcal{O}_{X} \rightarrow \mathcal{O}_{X}\left(3 \sigma-3 K_{B}\right) \oplus \mathcal{O}_{X}\left(2 \sigma-2 K_{B}\right) \oplus \mathcal{O}_{X}(\sigma) \rightarrow T_{\bar{\pi}}\right|_{X} \rightarrow 0,
\end{aligned}
$$

where $\bar{\pi}: \mathcal{A} \rightarrow B$ is the ambient space that the Calabi-Yau is embedded inside it. ${ }^{18}$ Now, by the last sequence above, one can show

$$
\pi_{*}\left(\left.T_{\bar{\pi}}\right|_{X} \otimes \mathcal{O}_{X}(-\sigma)\right)=K_{B}^{-3} \otimes\left(K_{B}^{2} \oplus \mathcal{O}_{B}\right) \oplus K_{B}^{-2} \oplus \mathcal{O}_{B}
$$

On the other hand, one can use the second sequence to show,

$$
R \pi_{*}\left(\left.T \mathcal{A}\right|_{X} \otimes \mathcal{O}_{X}(-\sigma)\right)=\pi_{*}\left(\left.T_{\bar{\pi}}\right|_{X} \otimes \mathcal{O}_{X}(-\sigma)\right) \oplus T B \otimes K_{B}[-1] .
$$

\footnotetext{
${ }^{18}$ Note that $\mathcal{A}$ is a $\mathbb{P}^{231}$ fibration.
} 
Finally the first short exact sequence, and the result above leads to ${ }^{19}$

$$
0 \rightarrow \pi_{*}\left(\left.T_{\bar{\pi}}\right|_{X} \otimes \mathcal{O}_{X}(-\sigma)\right) \rightarrow\left(\left.\pi_{*} \mathcal{N}\right|_{X} \otimes \mathcal{O}_{X}(-\sigma)\right) \rightarrow R^{1} \pi_{*}\left(T X \otimes \mathcal{O}_{X}(-\sigma)\right) \rightarrow T B \otimes K_{B} \rightarrow 0
$$

or, by considering the cokernel of the second map, we can rewrite this sequence as

$$
0 \rightarrow K_{B}^{-10} \otimes \mathcal{I}_{24 K^{2}} \rightarrow R^{1} \pi_{*}\left(T X \otimes \mathcal{O}_{X}(-\sigma)\right) \rightarrow T B \otimes K_{B} \rightarrow 0
$$

where, $\mathcal{I}_{24 K^{2}}$ is the ideal sheaf of a finite number of points given by the intersection of divisors $\left(-6 K_{B}\right) \cdot\left(-4 K_{B}\right)$. Now we can talk about the cohomologies of $T X \otimes \mathcal{O}_{X}(-\sigma)$ in (A.3). By the Leray spectral sequence,

$$
\left.E_{2}^{p, q}:=H^{p}\left(B, R^{q} \pi_{*}\left(T X \otimes \mathcal{O}_{X}(-\sigma)\right)\right) \Rightarrow H^{p+q}\left(X, T X \otimes \mathcal{O}_{X}(-\sigma)\right)\right)
$$

Since $R^{0} \pi_{*}\left(T X \otimes \mathcal{O}_{X}(-\sigma)\right)=0$ then

$$
\left.H^{p}\left(X, T X \otimes \mathcal{O}_{X}(-\sigma)\right)\right)=H^{p-1}\left(B, R^{1} \pi_{*}\left(T X \otimes \mathcal{O}_{X}(-\sigma)\right)\right)
$$

The cohomology long exact sequence of the equation (A.4) leads to,

$$
\begin{aligned}
0 & \rightarrow H^{0}\left(K_{B}^{-10}\right) \rightarrow H^{0}\left(R^{1} \pi_{*}\left(T X \otimes \mathcal{O}_{X}(-\sigma)\right)\right) \rightarrow H^{0}\left(T B \otimes K_{B}\right) \rightarrow \\
& \rightarrow H^{1}\left(K_{B}^{-10}\right) \rightarrow H^{1}\left(R^{1} \pi_{*}\left(T X \otimes \mathcal{O}_{X}(-\sigma)\right)\right) \rightarrow H^{1}\left(T B \otimes K_{B}\right) \rightarrow \\
& \rightarrow H^{2}\left(K_{B}^{-10}\right) \rightarrow H^{2}\left(R^{1} \pi_{*}\left(T X \otimes \mathcal{O}_{X}(-\sigma)\right)\right) \rightarrow H^{2}\left(T B \otimes K_{B}\right) \rightarrow 0
\end{aligned}
$$

By the ampleness assumption of $K_{B}^{-1}$ we expect $H^{i}\left(B, K_{B}^{-10}\right)=0$ for $i \neq 0$. So

$$
\begin{aligned}
& \left.H^{2}\left(X, T X \otimes \mathcal{O}_{X}(-\sigma)\right)\right)=H^{1}\left(R^{1} \pi_{*}\left(T X \otimes \mathcal{O}_{X}(-\sigma)\right)\right)=H^{1}\left(T B \otimes K_{B}\right)=H^{1,1}(B), \\
& \left.H^{3}\left(X, T X \otimes \mathcal{O}_{X}(-\sigma)\right)\right)=H^{2}\left(R^{1} \pi_{*}\left(T X \otimes \mathcal{O}_{X}(-\sigma)\right)\right)=H^{2}\left(T B \otimes K_{B}\right)=H^{0}\left(B, T B^{*}\right)
\end{aligned}
$$

At the end note that in general $h^{2}(X, T X)=h^{1,1}(X)=1+h^{1,1}(B)$, and also from (A.1) we can show $H^{2}\left(\left.T X\right|_{B}\right)=H^{2}\left(K_{B}\right) \oplus H^{2}(T B)=\mathbb{C} \oplus H^{2}(T B)$. Then to get the dimension of $H^{2}(T X)$ in (A.3) correct, all of the elements of $\left.H^{2}\left(X, T X \otimes \mathcal{O}_{X}(-\sigma)\right)\right)=H^{1,1}(B)$ should inject into $H^{2}(T X)$. Therefore we get a surjection (at least as long as $H^{1}\left(\mathcal{O}_{B}\right)=0$ ),

$$
H^{1}(T X) \rightarrow H^{1}\left(\left.T X\right|_{B}\right) \simeq H^{1}(T B) \rightarrow 0
$$

We conclude that $H^{1}(T B)$ is a subspace of the moduli space $H^{1}(T X)$ as expected.

\footnotetext{
${ }^{19}$ Note that $\pi_{*}\left(T X \otimes \mathcal{O}_{X}(-\sigma)\right)=0$ because $T X$ is a stable bundle, and one can show its restriction on generic fiber of $X$ is given by non-trivial extension of trivial bundles. Therefore $T X \otimes \mathcal{O}_{X}(-\sigma)$ doesn't have any global section on generic fibers. Naively, we may say $\pi_{*}\left(T X \otimes \mathcal{O}_{X}(-\sigma)\right)$ should be a torsion sheaf on $B$ (probably non-zero only on the discriminant curve of $X$ ), but since $\pi$ is flat and $T X \otimes \mathcal{O}_{X}(-\sigma)$ locally free, the pushforward $\pi_{*}\left(T X \otimes \mathcal{O}_{X}(-\sigma)\right)$ must be locally free, and hence should vanish everywhere.
} 


\section{B Type II and type III Fourier-Mukai calculations}

For completeness, we present some details of the Fourier-Mukai calculations as much as is necessary in this section. Following [33] we can compute the Chern characters of the Fourier-Mukai $\Phi(V)$ of V. In type II example, it is,

$$
C h(\Phi(V))=-[\mathcal{S}]+\left([\mathcal{S}] \cdot\left(\frac{C_{1}(B)}{2}\right)+\frac{1}{2} c_{3}(V)[f]\right)
$$

where $[\mathcal{S}]=\eta+n \sigma$. In type II example we chose $\eta=\eta_{0}+e_{1}$, and with this choice the algebraic equation of $[\mathcal{S}]$ will be reducible. Therefore the spectral line bundle $\mathcal{N}$ will be given as an extension,

$$
0 \longrightarrow i_{e_{1} *} \mathcal{L}_{1} \longrightarrow \mathcal{N} \longrightarrow i_{S_{0} *} \mathcal{L}_{2} \longrightarrow 0
$$

where $i_{e_{1}}$ and $i_{S_{0}}$ are respectively the inclusion morphisms of the surfaces $\pi^{*} e_{1}$ and $S_{0}$ with divisor class $n \sigma+\eta_{0}$. Now one can use,

$$
\Phi(V)=i_{S *} \mathcal{N}[-1] \Longrightarrow C h(\Phi(V))=-C h\left(i_{S *} \mathcal{N}\right)=-C h\left(i_{e_{1} *} \mathcal{L}_{1}\right)-C h\left(i_{S_{0} *} \mathcal{L}_{2}\right) .
$$

Next we can use the Grothendieck-Riemann-Rock to compute,

$$
\begin{aligned}
& C h\left(i_{e_{1} *} \mathcal{L}_{1}\right)=\left[e_{1}\right] \cdot\left(\frac{e^{c_{1}\left(\mathcal{L}_{1}\right)}}{\operatorname{Td}\left(\mathcal{O}\left(e_{1}\right)\right)}\right), \\
& C h\left(i_{S_{0} *} \mathcal{L}_{2}\right)=\left[S_{0}\right] \cdot\left(\frac{s^{c_{1}\left(\mathcal{L}_{2}\right)}}{\operatorname{Td}\left(\mathcal{O}\left(S_{0}\right)\right)}\right) .
\end{aligned}
$$

In order by order comparison of the formulas written above leads to the following result,

$$
c_{1}\left(\mathcal{L}_{2}\right)=\frac{1}{2}\left(c_{1}(B)-\left[\mathcal{S}_{0}\right]\right)+\lambda\left(n \sigma-\eta_{0}+n c_{1}(B)\right)+\lambda_{1} e_{1}
$$

A similar calculation for the type III example would lead to,

$C h(\Phi(V))=-\left[n \sigma+\eta+\pi^{*} \pi_{*}[C]\right]+\left[n \sigma+\eta+\pi^{*} \pi_{*}[C]\right] \cdot\left(\frac{c_{1}(B)}{2}-\frac{1}{2} \pi^{*} \pi_{*}[C]\right)+[C]+\ldots$

Again order by order comparison of this with the Chern character of the spectral sheaf over a smooth surface $i_{S *} \mathcal{N}$ shows that first of all, the divisor class of the spectral cover is

$$
[S]=n \sigma+\eta+\pi^{*} \pi_{*}[C]
$$

second Chern class of the line bundle $\mathcal{N}$ must be,

$$
c_{1}(\mathcal{N})=\frac{1}{2}\left(\left[S\left(V_{n}\right)\right]-c_{1}(B)\right)+\lambda\left(n \sigma-\eta+n c_{1}(B)\right)+\left(\frac{1}{n} \pi_{*}[C]-[C]\right) .
$$




\section{Computation details of "jumping" ext}

We show the calculation details about the "jumping" extension group used to stabilize complex structure moduli in subsection 3.1. The base $B$ of the elliptic Calabi-Yau threefold $X$ is defined in (3.13). The defining polynomial $p_{0}$ is a degree $(2,2)$ polynomial in $\left\{x_{0}, x_{1}\right\}$ and $\left\{y_{0}, y_{1}, y_{2}\right\}$. The coefficient of $p_{0}$ is a redundant description of the complex structure of $B$. We will extract the independent ones from them later.

The rank two extension bundle $V$ is introduced in (2.7) with extension group $\operatorname{Ext}\left(L, L^{\vee}\right)=H^{1}\left(X, L^{2}\right)$. If the threefold $X$ admits an elliptic fibration, this group can be expressed as

$$
H^{1}\left(X, L^{2}\right)=H^{0}\left(B, L_{1} \oplus L_{2}\right),
$$

where $L_{1}=O\left(D_{b}-C_{1}(B)\right)$ and $L_{2}=O\left(D_{b}+C_{1}(B)\right)$ are line bundles and $D_{b}$ is a divisor on the base. Thus, the Ext group of $V$ is a direct sum of $H^{0}\left(B, L_{1}\right)$ and $H^{0}\left(B, L_{2}\right)$. We will study the "jumping" of them in the following.

The line bundle cohomology on the base $B$ follows from a similar procedure discussed in subsection 2.1. As before, we can write the $L_{1}$ and $L_{2}$ in terms of the line bundles of the ambient space by Koszul sequence [37]. The associated long exact sequence is

$$
0 \rightarrow H^{0}(B, L) \rightarrow H^{1}\left(\mathcal{A}, N^{\vee} \otimes L_{\mathcal{A}}\right) \stackrel{p_{0}}{\rightarrow} H^{1}\left(\mathcal{A}, L_{\mathcal{A}}\right) \rightarrow H^{1}(B, L) \rightarrow 0 .
$$

Notice that, instead of $H^{1}\left(X, L^{2}\right)$, we are interested in the $H^{0}(B, L)$ here. For our example considered in section $3, L_{1}=O(-2,3)$ and $L_{2}=O(-2,5)$. From the equation (C.2), we find

$$
\begin{aligned}
& H^{0}\left(X, L_{1}\right)=\operatorname{Ker}\left[H^{1}(A, O(-4,1)) \stackrel{p_{0}}{\rightarrow} H^{1}(A, O(-2,3))\right] \\
& H^{0}\left(X, L_{2}\right)=\operatorname{Ker}\left[H^{1}(A, O(-4,3)) \stackrel{p_{0}}{\rightarrow} H^{1}(A, O(-2,5))\right]
\end{aligned}
$$

To determine the kernel, we express the cohomology of the source and the target in terms of their Bott-Borel-Weil polynomial representations. For the first one in equation (C.3), they are

$$
\begin{aligned}
& H^{1}\left(\mathbb{P}^{1} \times \mathbb{P}^{2}, O(-4,1)\right)=\left\{\frac{y_{0}}{x_{0}^{2}}, \frac{y_{1}}{x_{0}^{2}}, \ldots \frac{y_{0}}{x_{0} x_{1}}, \ldots \frac{y_{2}}{x_{1}^{2}}\right\} \\
& H^{1}\left(\mathbb{P}^{1} \times \mathbb{P}^{2}, O(-2,3)\right)=\left\{y_{0}^{3}, y_{0}^{2} y_{1}, \ldots y_{2}^{3}\right\}
\end{aligned}
$$

and $p_{0}$ is the degree $(2,2)$ defining polynomial. Note that the multiplication of $\frac{y_{0}}{x_{0} x_{1}}$ and $x_{0}^{2} y_{0}^{2}$ maps to zero in the target because the resulting monomials have the wrong degree and should not be considered as an appropriate element of the target. The same is for a map like $x_{1}^{2} y_{0}^{2}$. Thus, the most general form of the $p_{0}$ that have a non-trivial kernel is given by

$$
p_{0}=x_{1}^{2} P_{1}(y)+x_{2}^{2} P_{2}(y),
$$

where $P_{1}(y)$ and $P_{2}(y)$ are arbitrary degree 2 polynomials in $\left\{y_{0}, y_{1}, y_{2}\right\}$. There are three independent monomials with the denominator $x_{0} x_{1}$. Therefore, the dimension of the kernel "jumps" to 3 when the defining polynomial has the form in (C.7), and it vanishes when $p_{0}$ is a generic polynomial. By the same approach, we also compute the "jumping" of $H^{0}\left(X, L_{2}\right)$. The results are summarized in equation (3.17) that is used for complex structure moduli stabilization in section 3 . 


\section{Practical approach to identify reducible deformations}

In subsection 3.2, we find that the space of reducible deformations $M_{r}^{2}$ is generated by 5 out of 6 polynomials given in (3.35). There, we use the fact that polynomial deformation $\delta F_{2}$, if reducible, is equivalent to shifts of the coefficients in $F_{2}^{(0)}$. We will see why it is true and verify that $M_{r}^{2}$ contains all the possible reducible deformations. In addition, we will generalize the method introduced in subsection 3.2 to find reducible deformations of other initial polynomials.

Let's expand the initial $F_{2}^{(0)}$ defined in $(3.34)$ as

$$
F_{2}^{(0)}=\left(a u_{1}^{2}+b u_{1} u_{2}+c u_{2}^{2}\right) z_{1}^{2}+\left(d u_{1}+e u_{2}\right) z_{1} z_{2}+f z_{2}^{2} .
$$

where coefficients $a, b, c, d, e, f$ are given by

$$
\begin{array}{lll}
a=A_{1} B_{1}, & b=A_{1} B_{2}+A_{2} B_{1}, & c=A_{2} B_{2}, \\
d=A_{1} B_{3}+A_{3} B_{1}, & e=A_{2} B_{3}+A_{3} B_{2}, & f=A_{3} B_{3}
\end{array}
$$

with $A_{1}, A_{2}, A_{3}, B_{1}, B_{2}, B_{3}$ the coefficient of $F_{2}^{(0)}$ in the factored form. It means that given a polynomial with coefficients $a, b, c, d, e, f$, if one can find a set of $\left\{A_{i}\right\}$ and $\left\{B_{i}\right\}$ solves the equation (D.2), the polynomial is reducible. This is the reducible condition that will be used later. Obviously, $F_{2}^{(0)}$ is expressed by the following 6 monomials

$$
\left(u_{1}^{2} z_{1}^{2}, u_{1} u_{2} z_{1}^{2}, u_{2}^{2} z_{1}^{2}, u_{1} z_{1} z_{2}, u_{2} z_{1} z_{2}, z_{2}^{2}\right) .
$$

Thus, there are at most 6 polynomial deformations for $F_{2}^{(0)}$. Each of those is given by the deformation of a monomial. We will study which of these monomial deformations or their combinations $\delta F_{2}$ can keep $F_{2}^{(0)}+\delta F_{2}$ reducible.

Suppose we deform the initial $F_{2}^{(0)}$ by the first monomial $\delta a u_{1}^{2} z_{1}^{2}$. The coefficients of the deformed polynomial $F_{2}^{(0)}+\delta a u_{1}^{2} z_{1}^{2}$ becomes $\{a+\delta a, b, c, d, e, f\}$, which does not satisfy the reducible condition in (D.2). Thus, the deformed polynomial is not reducible. To restore the reducibility, one needs to adjust the coefficients $\left\{A_{i}\right\}$ and $\left\{B_{i}\right\}$ so that they give rise to the new parameters $\{a+\delta a, b, c, d, e, f\}$ via the condition (D.2). Since $a=A_{1} B_{1}$, it can be done by simply redefining $A_{1}$ to be $A_{1}^{\prime}=A_{1}+\frac{\delta a}{B_{1}}$. Now, $a+\delta a=A_{1}^{\prime} B_{1}$. To satisfy the reducible condition (D.2), one needs to replace the $A_{1}$ with $A_{1}^{\prime}$ in all other equations involving $A_{1}$, which are $b=A_{1}^{\prime} B_{2}+A_{2} B_{1}$ and $d=A_{1}^{\prime} B_{3}+A_{3} B_{1}$. The redefinition is equivalent to shift $b \rightarrow b+\left(\frac{B_{2}}{B_{1}}\right) \delta a$ and $d \rightarrow d+\left(\frac{B_{3}}{B_{1}}\right) \delta a$. That means, to preserve the reducibility, whenever we deform the $F$ by $\delta a u_{1}^{2} z_{1}^{2}$, we have to add the extra terms $\left(\frac{B_{2}}{B_{1}}\right) u_{1} u_{2} z_{1}^{2} \delta a$ and $\left(\frac{B_{3}}{B_{1}}\right) u_{1} z_{1} z_{2} \delta a$ as well. The reducible deformation after a redefinition of $A_{1}$ is

$$
e_{1}=u_{1}^{2} z_{1}^{2}+\left(\frac{B_{2}}{B_{1}}\right) u_{1} u_{2} z_{1}^{2}+\left(\frac{B_{3}}{B_{1}}\right) u_{1} z_{1} z_{2}
$$

which, by a factor of $B_{1}$, is the same deformations $e_{1}^{A}=B_{1} e_{1}$ as the one obtained by a shift of $A_{1}$ in (3.35).

Besides the redefinition of $A_{1}$, to absorb the deformation $\delta a u_{1}^{2} z_{1}^{2}$, one can also consider the redefinition of the $B_{1}$ as $B_{1}^{\prime}=B_{1}+\frac{\delta a}{A_{1}}$. From the relation (D.2), one has to redefine 
the $b$ and $d$ by shifting $b \rightarrow b+\left(\frac{A_{2}}{A_{1}}\right) \delta a$ and $d \rightarrow d+\left(\frac{A_{3}}{A_{1}}\right) \delta a$, which is given by

$$
e_{2}=u_{1}^{2} z_{1}^{2}+\left(\frac{A_{2}}{A_{1}}\right) u_{1} u_{2} z_{1}^{2}+\left(\frac{A_{3}}{A_{1}}\right) u_{1} z_{1} z_{2}
$$

Again, up to a factor of $A_{1}$, it is the same deformation obtained by a shift of $B_{1}$. Following the same analysis, we consider all 6 monomial deformations in (D.3). We find that the shifts of the coefficients can absorb all these deformations $\left\{A_{i}\right\}$ and $\left\{B_{i}\right\}$, which gives the same reducible deformations as ones in $M_{r}^{2}$.

The reducible deformations considered above are obtained by the redefinition of one coefficients. For example, $e_{1}$ and $e_{2}$ are generated by a shift of $A_{1}$ and $B_{1}$. One may suspect that can we get more reducible deformations by redefining $A_{1}$ and $B_{1}$ at the same time? For example, given the monomial deformation $\delta a u_{1}^{2} z_{1}^{2}$, one can absorb it by shifting the $A_{1}$ and $B_{1}$ at the same time by $\delta A_{1}=\frac{\delta a}{2 B_{1}}$ and $\delta B_{1}=\frac{\delta a}{2 A_{1}}$. It is easy to check that, to first-order, the reducible condition (D.2) is satisfied. In particular, $a+\delta a=\left(A_{1}+\delta A_{1}\right)\left(B_{1}+\delta B_{1}\right)$. The corresponding reducible deformations $E$ follow by shifting the $A_{1}$ and $B_{1}$ in all other equations in (D.2). One can show that $E=\frac{1}{2} e_{1}+\frac{1}{2} e_{2}$, which means that $E$ is included in $M_{r}^{2}$ and redefining multiple coefficients at the same time does not give new reducible deformations. Therefore, we verify that $M_{r}^{2}$ contains all the possible reducible deformations, and they are generated by the shifts of each coefficient in (3.34) independently.

We can generalize the above method to study the reducible deformations of a general initial $F_{2}^{(0)}$, which is a product of a polynomial with $m$ terms and a polynomial with $n$ terms like below

$$
F_{2}^{(0)}=\left(A_{1} m_{1}^{A}+A_{2} m_{2}^{A}+\ldots+A_{r} m_{r}^{A}\right)\left(B_{1} m_{1}^{B}+B_{2} m_{2}^{B}+\ldots+B_{s} m_{s}^{B}\right) .
$$

Here $\left\{m_{i}^{A}\right\}, i=1,2, \ldots, r$ and $\left\{m_{j}^{B}\right\}, j=1,2, \ldots, s$ are monomials and $\left\{A_{i}\right\}$ and $\left\{B_{j}\right\}$ are the corresponding coefficients. As before, the reducible deformations can be obtained by shifts of each coefficient in $\left\{A_{i}\right\}$ and $\left\{B_{j}\right\}$,

$$
\begin{array}{cc}
e_{1}^{A}=\left(B_{1} m_{1}^{B}+B_{2} m_{2}^{B}+\ldots+B_{s} m_{s}^{B}\right) m_{1}^{A}, & e_{1}^{B}=\left(A_{1} m_{1}^{A}+A_{2} m_{2}^{A}+\ldots+A_{r} m_{r}^{A}\right) m_{1}^{B} \\
e_{2}^{A}=\left(B_{1} m_{1}^{B}+B_{2} m_{2}^{B}+\ldots+B_{s} m_{s}^{B}\right) m_{2}^{A}, & e_{2}^{B}=\left(A_{1} m_{1}^{A}+A_{2} m_{2}^{A}+\ldots+A_{r} m_{r}^{A}\right) m_{2}^{B}, \\
\vdots & \vdots \\
e_{r}^{A}=\left(B_{1} m_{1}^{B}+B_{2} m_{2}^{B}+\ldots+B_{s} m_{s}^{B}\right) m_{r}^{A}, & e_{s}^{B}=\left(A_{1} m_{1}^{A}+A_{2} m_{2}^{A}+\ldots+A_{r} m_{r}^{A}\right) m_{s}^{B} .
\end{array}
$$

There are total $(r+s)$ such polynomials. As the equation (3.36), one can verify that these deformations satisfy the relation

$$
F_{2}^{(0)}+\left(A_{1} e_{1}^{A}+A_{2} e_{2}^{A}+\ldots+A_{r} e_{r}^{A}\right)=F_{2}^{(0)}+\left(B_{1} e_{1}^{B}+B_{2} e_{2}^{B}+\ldots+B_{s} e_{s}^{B}\right) .
$$

Thus, there are $(r+s-1)$ independent polynomials, and they form a basis of $M_{r}^{2}$.

Open Access. This article is distributed under the terms of the Creative Commons Attribution License (CC-BY 4.0), which permits any use, distribution and reproduction in any medium, provided the original author(s) and source are credited. 


\section{References}

[1] P. Candelas, G.T. Horowitz, A. Strominger and E. Witten, Vacuum configurations for superstrings, Nucl. Phys. B 258 (1985) 46 [INSPIRE].

[2] M.B. Green, J.H. Schwarz and E. Witten, Superstring theory. Volume 2: loop amplitudes, anomalies and phenomenology, Cambridge Univ. Pr., Cambridge, U.K. (1987).

[3] V. Braun, Y.-H. He, B.A. Ovrut and T. Pantev, A heterotic standard model, Phys. Lett. B 618 (2005) 252 [hep-th/0501070] [INSPIRE].

[4] V. Braun, Y.-H. He, B.A. Ovrut and T. Pantev, A standard model from the $E_{8} \times E_{8}$ heterotic superstring, JHEP 06 (2005) 039 [hep-th/0502155] [INSPIRE].

[5] V. Braun, Y.-H. He, B.A. Ovrut and T. Pantev, Vector bundle extensions, sheaf cohomology, and the heterotic standard model, Adv. Theor. Math. Phys. 10 (2006) 525 [hep-th/0505041] [INSPIRE].

[6] V. Bouchard and R. Donagi, An SU(5) heterotic standard model, Phys. Lett. B 633 (2006) 783 [hep-th/0512149] [INSPIRE].

[7] L.B. Anderson, J. Gray, Y.-H. He and A. Lukas, Exploring positive monad bundles and a new heterotic standard model, JHEP 02 (2010) 054 [arXiv:0911.1569] [INSPIRE].

[8] V. Braun, P. Candelas, R. Davies and R. Donagi, The MSSM spectrum from $(0,2)$-deformations of the heterotic standard embedding, JHEP 05 (2012) 127 [arXiv: 1112.1097] [INSPIRE].

[9] L.B. Anderson, J. Gray, A. Lukas and E. Palti, Two hundred heterotic standard models on smooth Calabi-Yau threefolds, Phys. Rev. D 84 (2011) 106005 [arXiv:1106.4804] [INSPIRE].

[10] L.B. Anderson, J. Gray, A. Lukas and E. Palti, Heterotic line bundle standard models, JHEP 06 (2012) 113 [arXiv: 1202.1757] [INSPIRE].

[11] L.B. Anderson, A. Constantin, J. Gray, A. Lukas and E. Palti, A comprehensive scan for heterotic SU(5) GUT models, JHEP 01 (2014) 047 [arXiv: 1307.4787] [INSPIRE].

[12] L.B. Anderson, J. Gray, A. Lukas and B. Ovrut, Stabilizing the complex structure in heterotic Calabi-Yau vacua, JHEP 02 (2011) 088 [arXiv:1010.0255] [INSPIRE].

[13] L.B. Anderson, J. Gray, A. Lukas and B. Ovrut, The Atiyah class and complex structure stabilization in heterotic Calabi-Yau compactifications, JHEP 10 (2011) 032 [arXiv: 1107.5076] [INSPIRE].

[14] L.B. Anderson, J. Gray, A. Lukas and B. Ovrut, Stabilizing all geometric moduli in heterotic Calabi-Yau vacua, Phys. Rev. D 83 (2011) 106011 [arXiv:1102.0011] [InSPIRE].

[15] L.B. Anderson, J. Gray, A. Lukas and B. Ovrut, Vacuum varieties, holomorphic bundles and complex structure stabilization in heterotic theories, JHEP 07 (2013) 017 [arXiv:1304.2704] [INSPIRE].

[16] J. Gray and J. Wang, Jumping spectra and vanishing couplings in heterotic line bundle standard models, JHEP 11 (2019) 073 [arXiv:1906.09373] [INSPIRE].

[17] E.R. Sharpe, Kähler cone substructure, Adv. Theor. Math. Phys. 2 (1999) 1441 [hep-th/9810064] [INSPIRE].

[18] L.B. Anderson, J. Gray, A. Lukas and B. Ovrut, The edge of supersymmetry: stability walls in heterotic theory, Phys. Lett. B 677 (2009) 190 [arXiv:0903.5088] [InSPIRE]. 
[19] L.B. Anderson, J. Gray, A. Lukas and B. Ovrut, Stability walls in heterotic theories, JHEP 09 (2009) 026 [arXiv: 0905.1748] [INSPIRE].

[20] L.B. Anderson, J. Gray and B. Ovrut, Yukawa textures from heterotic stability walls, JHEP 05 (2010) 086 [arXiv: 1001.2317] [INSPIRE].

[21] L.B. Anderson, J. Gray and B.A. Ovrut, Transitions in the web of heterotic vacua, Fortsch. Phys. 59 (2011) 327 [arXiv: 1012.3179] [INSPIRE].

[22] M.F. Atiyah, Complex analytic connections in fibre bundles, Trans. Amer. Math. Soc. 85 (1957) 181.

[23] M. Gross, A finiteness theorem for elliptic Calabi-Yau threefolds, alg-geom/9305002 [INSPIRE].

[24] A. Constantin, J. Gray and A. Lukas, Hodge numbers for all CICY quotients, JHEP 01 (2017) 001 [arXiv: 1607.01830] [INSPIRE].

[25] L.B. Anderson, X. Gao, J. Gray and S.-J. Lee, Tools for CICYs in F-theory, JHEP 11 (2016) 004 [arXiv : 1608.07554] [inSPIRE].

[26] L.B. Anderson, X. Gao, J. Gray and S.-J. Lee, Fibrations in CICY threefolds, JHEP 10 (2017) 077 [arXiv : 1708.07907] [inSPIRE].

[27] L.B. Anderson, J. Gray and B. Hammack, Fibrations in non-simply connected Calabi-Yau quotients, JHEP 08 (2018) 128 [arXiv: 1805.05497] [INSPIRE].

[28] Y.-C. Huang and W. Taylor, On the prevalence of elliptic and genus one fibrations among toric hypersurface Calabi-Yau threefolds, JHEP 03 (2019) 014 [arXiv:1809.05160] [INSPIRE].

[29] Y.-C. Huang and W. Taylor, Mirror symmetry and elliptic Calabi-Yau manifolds, JHEP 04 (2019) 083 [arXiv: 1811.04947] [INSPIRE].

[30] Y.-C. Huang and W. Taylor, Fibration structure in toric hypersurface Calabi-Yau threefolds, JHEP 03 (2020) 172 [arXiv: 1907.09482] [InSPIRE].

[31] R. Friedman, J. Morgan and E. Witten, Vector bundles and F-theory, Commun. Math. Phys. 187 (1997) 679 [hep-th/9701162] [INSPIRE].

[32] R. Friedman, J.W. Morgan and E. Witten, Vector bundles over elliptic fibrations, alg-geom/9709029 [INSPIRE].

[33] L.B. Anderson, X. Gao and M. Karkheiran, Extending the geometry of heterotic spectral cover constructions, Nucl. Phys. B 956 (2020) 115003 [arXiv: 1912.00971] [INSPIRE].

[34] L.B. Anderson, H. Feng, X. Gao and M. Karkheiran, Heterotic/heterotic and heterotic/F-theory duality, Phys. Rev. D 100 (2019) 126014 [arXiv:1907.04395] [INSPIRE].

[35] A.P. Braun, A. Collinucci and R. Valandro, G-flux in F-theory and algebraic cycles, Nucl. Phys. B 856 (2012) 129 [arXiv:1107.5337] [INSPIRE].

[36] A.P. Braun and R. Valandro, $G_{4}$ flux, algebraic cycles and complex structure moduli stabilization, JHEP 01 (2021) 207 [arXiv:2009.11873] [INSPIRE].

[37] R. Hartshorne, Algebraic geometry, Springer-Verlag, New York, NY, U.S.A. (1977).

[38] S.K. Donaldson, Anti self-dual Yang-Mills connections over complex algebraic surfaces and stable vector bundles, Proc. London Math. Soc. s3-50 (1985) 1. 
[39] K. Uhlenbeck and S.-T. Yau, On the existence of Hermitian-Yang-Mills connections in stable vector bundles, Commun. Pure Appl. Math. 39 (1986) S257.

[40] K. Kodaira, Complex manifolds and deformation of complex structures, Springer, Berlin, Heidelberg, Germany (2005).

[41] S. Kobayashi, Differential geometry of complex vector bundles, Iwanami Shoten, Tokyo, Japan (1987).

[42] M. Kuranishi, Deformations of compact complex manifolds, in Seminaire de mathematiques superieures, no. 39 (ete 1969), Les Presses de l'Universite de Montreal, Montreal, QC, Canada (1971).

[43] S. Donaldson and R. Friedman, Connected sums of self-dual manifolds and deformations of singular spaces, Nonlinearity 2 (1989) 197.

[44] M. Gabella, Y.-H. He and A. Lukas, An abundance of heterotic vacua, JHEP 12 (2008) 027 [arXiv:0808.2142] [INSPIRE].

[45] C. Vafa, Evidence for F-theory, Nucl. Phys. B 469 (1996) 403 [hep-th/9602022] [InSPIRE].

[46] D.R. Morrison and C. Vafa, Compactifications of F-theory on Calabi-Yau threefolds. 1, Nucl. Phys. B 473 (1996) 74 [hep-th/9602114] [INSPIRE].

[47] D.R. Morrison and C. Vafa, Compactifications of F-theory on Calabi-Yau threefolds. 2, Nucl. Phys. B 476 (1996) 437 [hep-th/9603161] [INSPIRE].

[48] S. Gukov, C. Vafa and E. Witten, CFT's from Calabi-Yau four folds, Nucl. Phys. B 584 (2000) 69 [Erratum ibid. 608 (2001) 477] [hep-th/9906070] [INSPIRE].

[49] L.B. Anderson, J. Gray, A. Lukas and J. Wang, Chern-Simons invariants and heterotic superpotentials, JHEP 09 (2020) 141 [arXiv:2006.03082] [INSPIRE].

[50] L.B. Anderson, F. Apruzzi, X. Gao, J. Gray and S.-J. Lee, A new construction of Calabi-Yau manifolds: generalized CICYs, Nucl. Phys. B 906 (2016) 441 [arXiv:1507.03235] [InSPIRE].

[51] R. Donagi and M. Wijnholt, Model building with F-theory, Adv. Theor. Math. Phys. 15 (2011) 1237 [arXiv: 0802 .2969] [inSPIRE].

[52] C. Beasley, J.J. Heckman and C. Vafa, GUTs and exceptional branes in F-theory - I, JHEP 01 (2009) 058 [arXiv:0802.3391] [inSPIRE].

[53] R. Donagi and M. Wijnholt, Higgs bundles and UV completion in F-theory, Commun. Math. Phys. 326 (2014) 287 [arXiv: 0904.1218] [INSPIRE]. 